\title{
Ionized outflows in local luminous AGN: what are the real densities and outflow rates?
}

\author{
R. Davies, ${ }^{1}$ D. Baron, ${ }^{2}$ T. Shimizu, ${ }^{1}$ H. Netzer, ${ }^{2}$ L. Burtscher, ${ }^{3}$ P. T. de Zeeuw, ${ }^{1,3}$ \\ R. Genzel, ${ }^{1}$ E.K.S. Hicks ${ }^{4}$ M. Koss,${ }^{5}$ M.-Y. Lin, ${ }^{6}$ D. Lutz,${ }^{1}$ W. Maciejewski, ${ }^{7}$ \\ F. Müller-Sánchez ${ }^{8}$ G. Orban de Xivry, ${ }^{9}$ C. Ricci, ${ }^{10}$ R. Riffel, ${ }^{11}$ R.A. Riffel, ${ }^{12}$ \\ D. Rosario, ${ }^{13}$ M. Schartmann, ${ }^{1}$ A. Schnorr-Müller, ${ }^{11}$ J. Shangguan, ${ }^{1}$ A. Sternberg, ${ }^{2}$ \\ E. Sturm, ${ }^{1}$ T. Storchi-Bergmann, ${ }^{11}$ L. Tacconi, ${ }^{1}$ and S. Veilleux ${ }^{14}$ \\ ${ }_{1}$ Max-Planck-Institut für extraterrestrische Physik, Postfach 1312, 85741, Garching, Germany \\ 2 School of Physics and Astronomy, Tel-Aviv University, Tel Aviv 69978, Israel \\ ${ }^{3}$ Leiden Observatory, Leiden University, PO Box 9513, 2300 RA, Leiden, the Netherlands \\ ${ }^{4}$ Department of Physics 83 Astronomy, University of Alaska Anchorage, AK 99508-4664, USA \\ 5 Eureka Scientific, 2452 Delmer Street Suite 100, Oakland, CA 94602-3017, USA \\ ${ }^{6}$ Institute of Astronomy and Astrophysics, Academia Sinica, Roosevelt Rd, Taipei 10617, Taiwan \\ 7 Astrophysics Research Institute, Liverpool John Moores University, IC2 Liverpool Science Park, 146 Brownlow Hill, L3 5RF, UK \\ 8 Physics Department, University of Memphis, Memphis, TN 38152, USA \\ 9 Space Sciences, Technologies, and Astrophysics Research Institute, Université de Liège, 4000 Sart Tilman, Belgium \\ ${ }^{10}$ Instituto de Astrofísica, Facultad de Física, Pontificia Universidad Católica de Chile, Casilla 306, Santiago 22, Chile \\ 11 Departamento de Astronomia, Universidade Federal do Rio Grande do Sul, IF, CP 15051, 91501-970 Porto Alegre, RS, Brazil \\ 12 Departamento de Física, Universidade Federal de Santa Maria, 97105-900 Santa Maria, RS, Brazil \\ 13 Department of Physics, Durham University, South Road, Durham, DH1 3LE, UK \\ 14 Department of Astronomy and Joint Space-Science Institute, University of Maryland, College Park, MD 20742-2421, USA
}

Accepted XXX. Received YYY; in original form ZZZ

\begin{abstract}
We report on the determination of electron densities, and their impact on the outflow masses and rates, measured in the central few hundred parsecs of 11 local luminous active galaxies. We show that the peak of the integrated line emission in the AGN is significantly offset from the systemic velocity as traced by the stellar absorption features, indicating that the profiles are dominated by outflow. In contrast, matched inactive galaxies are characterised by a systemic peak and weaker outflow wing. We present three independent estimates of the electron density in these AGN, discussing the merits of the different methods. The electron density derived from the [SII] doublet is significantly lower than than that found with a method developed in the last decade using auroral and transauroral lines, as well as a recently introduced method based on the ionization parameter. The reason is that, for gas photoionized by an AGN, much of the [SII] emission arises in an extended partially ionized zone where the implicit assumption that the electron density traces the hydrogen density is invalid. We propose ways to deal with this situation and we derive the associated outflow rates for ionized gas, which are in the range $0.001-0.5 \mathrm{M}_{\odot} \mathrm{yr}^{-1}$ for our AGN sample. We compare these outflow rates to the relation between $\dot{M}_{\text {out }}$ and $L_{A G N}$ in the literature, and argue that it may need to be modified and rescaled towards lower mass outflow rates.
\end{abstract}

Key words: Galaxies: active - Galaxies: ISM - Galaxies: nuclei - Galaxies: Seyfert

\section{INTRODUCTION}

The evidence that outflows, whether driven by star formation or AGN, play a fundamental role in the evolution of galaxies is now long undisputed (Veilleux et al. 2005; Fabian 2012; Heckman \& Best 2014; Somerville \& Davé 2015; King
$\&$ Pounds 2015). However, the amount of gas in each phase of the outflow, and whether the outflow escapes the host galaxy or if it has a significant impact on the global star formation rate, are not yet firmly established (Veilleux et al. 2020). As emphasized by Harrison et al. (2018), for ionized 
outflows, a large part of the uncertainty is directly linked to the density of gas in the outflow. The reason is straightforward to show because, at a fixed (i.e. the observed) line luminosity, the derived mass of ionized gas, and hence outflow rate, is inversely proportional to the adopted density. The line luminosity is $L_{\text {line }}=\gamma_{\text {line }} n_{e} n_{p} V f$ where $\gamma_{\text {line }}$ is the appropriate volume emissivity, $n_{e} \sim n_{p}$ is the electron or equivalently ion density, $V$ is the volume, and $f$ the filling factor. An additional implicit assumption is that $n_{e} \sim n_{H}$, that is the clouds are fully ionized. In this case, the mass of ionized gas in that volume is $M_{\text {out }}=\mu m_{H} n_{p} V f$ where $\mu m_{H}$ is the effective atomic mass. Together these show that the dependencies of the derived ionized gas mass are $M_{\text {out }} \propto L_{\text {line }} /\left(\gamma_{\text {line }} n_{e}\right)$ and equivalently the dependencies for the outflow rate are $\dot{M}_{\text {out }} \propto L_{\text {line }} v_{\text {out }} /\left(\gamma_{\text {line }} n_{e} r_{\text {out }}\right)$. Thus, a reliable assessment of the density in the outflow, that is appropriate to the spatial scales being measured, is an essential ingredient for deriving the outflow mass and rate.

In the literature, a wide range of different densities (either assumed or measured), covering several orders of magnitude, have been used when deriving quantities related to ionized outflows driven by active galactic nuclei (AGN). These include, at low and high redshift: $100 \mathrm{~cm}^{-3}$ (Liu et al. 2013; Riffel et al. 2013; Harrison et al. 2014; Kakkad et al. 2016; Rupke et al. 2017); $200 \mathrm{~cm}^{-3}$ (Fiore et al. 2017); $500 \mathrm{~cm}^{-3}$ (Storchi-Bergmann et al. 2010; Carniani et al. 2015; Riffel et al. 2015); $1000-1500 \mathrm{~cm}^{-3}$ (Schnorr-Müller et al. 2016b; Perna et al. 2017; Förster Schreiber et al. 2019; Shimizu et al. 2019); $5000 \mathrm{~cm}^{-3}$ (Müller-Sánchez et al. 2011); and in some instances densities of $10^{4}-10^{5} \mathrm{~cm}^{-3}$ have been reported (Holt et al. 2011; Rose et al. 2018; Santoro et al. 2018; Baron \& Netzer 2019). Among this plethora of values, lower densities are often adopted or measured for larger scales of $1-10 \mathrm{kpc}$, while the higher densities apply to smaller $0.1-1 \mathrm{kpc}$ scales. This tendency is also reflected in spatially resolved studies (e.g. Baron et al. 2018; Freitas et al. 2018; Kakkad et al. 2018; Shimizu et al. 2019; Do Nascimento et al. 2019) which tend to show that $n_{e}$ decreases with radius.

In this paper, we make use of the high quality spectroscopic data available for the LLAMA (Local Luminous AGN with Matched Analogues) survey (Davies et al. 2015) to derive electron densities in the outflowing gas. These are then used to estimate the outflow rates, which are compared to well known relations between AGN luminosity and outflow rate. The paper begins with a description of the sample and observations in Sec. 2, together with estimates of the systemic velocity and a discussion of how the stellar continuum is subtracted. In Sec. 3 we argue, based on the line ratios and profiles, that the entire line profile in these AGNs is dominated by outflow, and any systemic component is subdominant. Because of this, when deriving densities, we integrate over the complete emission lines. The outflow densities are derived using three independent methods, which are summarized in Sec. 4. They are the standard method of the [SII] $\lambda 6716 / 6731$ doublet ratio; a method developed by Holt et al. (2011) which makes use of auroral and transauroral lines in the ratios $[\mathrm{SII}] \lambda(6716+6731) /[\mathrm{SII}] \lambda(4069+4076)$ and $[\mathrm{OII}] \lambda(3726+3729) /[\mathrm{OII}] \lambda(7320+7331)$; and a method recently introduced by Baron \& Netzer (2019) that is based on the definition of the ionization parameter. We discuss the merits of the various methods and present the densities de- rived from our sample of AGN for each of them. Using the density measure that we argue is most appropriate, in Section 5 we assess whether the derived outflow rates extend the lower luminosity end of the $L_{A G N}-\dot{M}_{\text {out }}$ relation proposed by Fiore et al. (2017). We finish with our conclusions in Section 6.

\section{SAMPLE AND OBSERVATIONS}

\subsection{Sample}

We make use of the LLAMA sample of active and inactive galaxies. Davies et al. (2015) provide the rationale for the sample, and a detailed description of its selection. The key aspect is that these are taken from the all-sky flux limited 14-195 keV 58-month Swift BAT survey (Baumgartner et al. 2013) in such a way as to create a volume limited sample of active galaxies that is as unbiased as possible, for detailed study using optical spectroscopy and adaptive optics integral field near-infrared spectroscopy. The sole selection criteria were $z<0.01$ (corresponding to a distance of $\sim 40 \mathrm{Mpc}$ ), $\log L_{14-195 \mathrm{keV}}\left[\mathrm{erg} \mathrm{s}^{-1}\right]>42.5$ (using redshift distance), and $\delta<15^{\circ}$ so that they are observable from the VLT. This yielded 20 AGN. A set of inactive galaxies were selected to match them in terms of host galaxy type, mass (using H-band luminosity as a proxy), inclination, presence of a bar, and distance. Although small, this volume limited sample is sufficient for detailed studies of emission line ratios, the molecular and ionized gas kinematics and distributions, as well as the stellar kinematics and populations, in the nuclear and circumnuclear regions. And the ability to compare the results to a matched sample of inactive galaxies has been essential in many of the studies so far, including the analysis presented here. These studies include: the physical properties of, and extinction to, the broad line region (Schnorr-Müller et al. 2016a); the respective roles of host galaxy and environment in fuelling AGN (Davies et al. 2017); the molecular gas content and depletion time on kiloparsec scales (Rosario et al. 2018); the nuclear stellar population and kinematics (Lin et al. 2018); the black hole masses and location in the $M_{B H}-\sigma *$ plane (Caglar et al. 2020); and the nuclear star formation histories (Burtscher et al. 2020).

For this paper, we have limited the subsample of active galaxies to include only Sy 1.8-2 types, i.e. the 11 objects without too prominent broad emission lines. The purpose of doing so is to avoid uncertainties in the measurement of emission lines with complex profiles that are superimposed on the very strong and sometimes irregular broad line emission. For the analysis using the auroral and transauroral lines, we had to limit the AGN further, omitting those for which the full set of necessary [SII] and [OII] lines could not be measured reliably (ESO 021-G004, NGC 1365, and NGC 7172). Because, as shown later, these are the 3 objects with the faintest line fluxes, excluding them does not introduce a line ratio bias. The subsample of inactive galaxies with Xshooter observations contains 18 objects. We exclude three - NGC 1315, NGC 2775, and NGC 5845 - which have no measurable line emission. Thus, there are 11 active and 15 inactive galaxies in the sample analysed in this paper. 
Table 1. Summary of Observations

\begin{tabular}{|c|c|c|c|c|}
\hline Object & type $^{a}$ & $v_{\text {sys }}\left(\mathrm{km} \mathrm{s}^{-1}\right)^{b}$ & \# obs & dates $^{c}$ \\
\hline ESO 137-G034 & Sy 2 & 2758 & 3 & $2015.05 .18,2015.05 .20,2015.06 .23$ \\
\hline MCG-05-23-016 & Sy 1.9 & 2498 & 2 & 2014.01 .21 \\
\hline NGC 2110 & Sy $2(1 \mathrm{~h})$ & 2318 & 2 & 2013.11 .24 \\
\hline NGC 2992 & Sy 1.8 & 2330 & 2 & $2014.02 .25,2014.02 .26$ \\
\hline NGC 3081 & Sy $2(1 \mathrm{~h})$ & 2380 & 2 & 2014.02 .19 \\
\hline NGC 5506 & Sy 2 (1i) & 1940 & 1 & 2016.03 .03 \\
\hline NGC 5728 & Sy 2 & 2791 & 2 & $2015.05 .12,2015.07 .15$ \\
\hline NGC 7582 & Sy 2 (1i) & 1589 & 2 & 2016.07.26, 2016.08.08 \\
\hline ESO 021-G004 & Sy 2 & 2847 & 1 & 2016.08 .01 \\
\hline NGC 1365 & Sy 1.8 & 1529 & 2 & 2013.12 .10 \\
\hline NGC 7172 & Sy 2 (1i) & 2567 & 2 & 2015.08.11 \\
\hline ESO 093-G003 & inactive & 1817 & 2 & 2014.01.21, 2014.03.20 \\
\hline ESO 208-G021 & inactive & 1068 & 2 & 2013.12.11, 2014.01.21 \\
\hline NGC 0718 & inactive & 1751 & 1 & 2015.12 .04 \\
\hline NGC 1079 & inactive & 1468 & 1 & 2013.11 .22 \\
\hline NGC 1947 & inactive & 1191 & 2 & $2013.12 .22,2014.02 .07$ \\
\hline NGC 3175 & inactive & 1099 & 2 & 2014.03.08, 2013.03.09 \\
\hline NGC 3351 & inactive & 777 & 2 & 2014.02 .20 \\
\hline NGC 3717 & inactive & 1741 & 2 & 2014.03 .21 \\
\hline NGC 3749 & inactive & 2743 & 2 & $2014.03 .21,2014.03 .31$ \\
\hline NGC 4224 & inactive & 2645 & 1 & 2015.05 .12 \\
\hline NGC 4254 & inactive & 2445 & 1 & 2016.06 .01 \\
\hline NGC 5037 & inactive & 1913 & 2 & $2015.05 .12,2016.02 .03$ \\
\hline NGC 5921 & inactive & 1502 & 2 & $2015.06 .15,2015.06 .23$ \\
\hline NGC 7727 & inactive & 1828 & 1 & 2015.08 .08 \\
\hline IC 4653 & inactive & 1528 & 2 & $2015.05 .18,2016.06 .03$ \\
\hline
\end{tabular}

a Classifications are taken from Davies et al. (2015) and references therein; 1i indicates the broad lines have been observed in the near-infrared, and $1 \mathrm{~h}$ that hidden broad lines have been detected via polarisation measurements.

${ }^{b}$ Systemic velocity is as derived in this work from fitting the CaII triplet absorption lines (except for NGC 5506 and NGC 1365 , for which the NaI D lines were used because the CaII was filled with Paschen series emission lines). It is the observed velocity, without any corrections applied. Uncertainty is typically $2 \mathrm{~km} \mathrm{~s}^{-1}$.

${ }^{c}$ See Burtscher et al. (2020) for details.
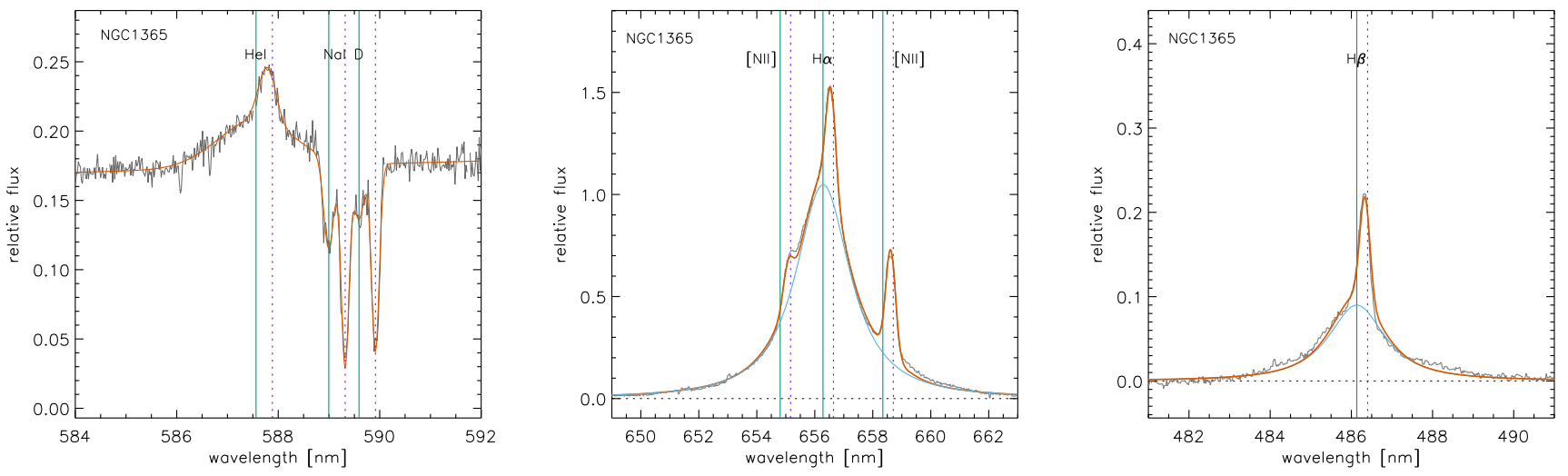

Figure 1. Spectral segments and fits to features in NGC1365, the most complex of our AGN to fit. The vertical green lines trace the systemic velocity while the dotted purple lines trace the outflow. Left: the region around the Na I D absorption, showing that there are two pairs of lines. The weaker absorption (centered at the same velocity as the broad line region) traces systemic, while the redshifted absorption (centered at the same velocity as the narrow emission lines) traces the outflow. Redshifted absorption tracing outflow is possible for the receding bicone projected against an extended stellar continuum. Centre: the region around the H $\alpha$ line. The broad line has been fit with a Moffat function, with its peak fixed to the velocity of the systemic Na I D absorption. The narrow emission lines are clearly redshifted with respect to the broad line. Right: the region around the $\mathrm{H} \beta$ line. Again, the broad $\mathrm{H} \beta$ is clearly centered at a different velocity to the narrow emission lines. 


\subsection{Observations and Data Reduction}

Details of the observations, data reduction, and spectral extraction, for all these objects are given in Burtscher et al. (2020). We have simply re-used those spectra. Although NGC 5506 was not included in their analysis, the data were processed in the same way and at the same time. We list in Table 1 the number of observations that were performed for each object, with their dates. Very briefly, we used the integral field mode of Xshooter and integrated for approximately $1 \mathrm{hr}$ for each observation of each source. The standard pipeline was used to process the data, and a 1D spectrum was extracted in a $1.8^{\prime \prime} \times 1.8^{\prime \prime}$ aperture from the UVB and VIS arms. The location of the extraction aperture on the object was determined in the NIR arm (although those data are not used in the analysis presented here), which allows the best centering because it is least affected by extinction and, for the AGN, includes bright non-stellar continuum. For the UVB and VIS arms, the extraction aperture location was adjusted with respect to the NIR according to the mean differential atmospheric refraction during the observation. Observations of the telluric and flux calibrator stars confirmed the validity of this approach. Flux calibration, and correction of telluric absorption, were performed on the extracted 1D spectra. Finally, the calibrated 1D spectra from the UVB and VIS arms were merged by scaling the overlapping region to provide a consistent flux calibration over $300 \mathrm{~nm}$ to $1.0 \mu \mathrm{m}$. The spectral resolution across this range is $R>8000$.

For each object, the resulting spectra from each observation were analysed separately in order to provide independent estimates of each measured property. By doing this, we are able to confirm that the uncertainties derived for these properties are consistent with the differences between the values measured from each spectrum. When more than one spectrum is available for an object, the resulting values reported in this paper are the weighted mean, with the associated uncertainty on that mean.

\subsection{Systemic Velocities}

A measure of each object's systemic velocity, $v_{\text {sys }}$, that is independent of the emission lines is an important aspect of the analysis presented here. For all except two of the objects, we have used the CaII triplet lines since these provide a clean and robust measurement of the stellar velocity in the aperture. We have fit a Gaussian profile to each of the lines at $8498 \AA, 8542 \AA$, and $8662 \AA$, using a local estimate of the continuum. The routine we used, mpfitpeak (Markwardt 2009), provides an estimate of the uncertainties. We confirmed that the uncertainties were consistent with the variation between the velocities determined for the three lines, and then combined the velocities with a weighted mean. The uncertainty on the mean was typically about $2 \mathrm{~km} \mathrm{~s}^{-1}$, and can therefore be considered negligible with respect to the velocity offsets of the emission lines discussed in Sec. 3.

In the case of NGC 5506 and NGC 1365, the CaII features were filled with strong Paschen H I emission. Instead, for these two objects we used the Na I D lines at $5890 \AA$ and $5896 \AA$ to estimate $v_{\text {sys }}$. Because they are partially blended in our spectrum, we fit them simultaneously using the routine mpfitfun (Markwardt 2009). As before, uncertainties were provided by the routine, and correspond to $7 \mathrm{~km} \mathrm{~s}^{-1}$. We note that in general the NaI D lines can have a contribution from a neutral outflow, which may bias our estimate of the systemic velocity (Rupke et al. 2005a,b). And indeed for NGC 1365 this complication was realised due to the presence of two pairs of $\mathrm{NaI} \mathrm{D}$ lines separated by $165 \mathrm{~km} \mathrm{~s}^{-1}$, as well as broad He I at $5876 \AA$, as can be seen in Fig. 1. Determining which of these traces the systemic stellar population and which is due to outflow is coupled to the fact that, when examining the spectral regions near $\mathrm{H} \alpha$ and $\mathrm{H} \beta$, the peak of the broad line is offset from the peak of the narrow line (see also Schnorr-Müller et al. 2016a). In terms of velocity, it is the component at the shorter wavelength that matches the broad line, indicating that the stellar population and central massive black hole trace the same potential. It is also apparent from Fig. 1 that the Na I D absorption at longer wavelengths approximately matches the peak of the narrow emission lines, indicating a likely association between outflowing neutral and ionized gas. A detailed analysis of such an asssociation in a post starburst $\mathrm{E}+\mathrm{A}$ galaxy using spatially resolved data is given by Baron et al. (2020). That the absorption can be both redshifted and outflowing is possible if it is tracing the receding bicone against an extended stellar continuum.

The procedures above yield also the stellar velocity dispersion. Within the uncertainties of the small numbers analysed here, the distributions for both the active and inactive galaxies are the same, with values for individual objects typically in the range $\sigma_{*} \sim 100-200 \mathrm{~km} \mathrm{~s}^{-1}$. In all cases the measured dispersion is at least a factor 3 greater than the instrumental broadening (more typically a factor 6-12), and so any correction for that will have a negligible effect.

The systemic velocities we measure (without any correction to heliocentric) are given in Table 1. Most of these are consistent with the values reported in the literature by the NASA/IPAC Extragalactic Database (NED). However, there were five galaxies where the difference exceeded $100 \mathrm{~km} \mathrm{~s}^{-1}$. We have checked these in order to confirm our measurements as follows:

NGC 5506 There are a large number of redshift measurements in NED, typically giving $1800-1850 \mathrm{~km} \mathrm{~s}^{-1}$ based on optical emission lines. Remarkably, Van den Bosch et al. (2015) quote $1750 \mathrm{~km} \mathrm{~s}^{-1}$ based on fitting the stellar continuum. However, Burtscher et al. (2020) found it is very difficult to get a good global fit to the stellar continuum due to the numerous strong and broad lines, and had to excluded this object from their analysis. For our measurement, we have instead searched for a well-defined narrow stellar feature that can be robustly measured. That the resulting velocity of $1940 \mathrm{~km} \mathrm{~s}^{-1}$ matches a dip in the emission line profile (which we argue later represents the transition between the approaching and receding sides of bicone outflow) gives us confidence that this is a reliable measurement. The peaks of the emission lines are blueshifted with respect to this, and fitting them with a single Gaussian yields $1810 \mathrm{~km} \mathrm{~s}^{-1}$, fully consistent with the published values.

ESO 021-G004 The only redshift in NED is $2960 \mathrm{~km} \mathrm{~s}^{-1}$ from an HI measurement. The HI source was matched to ESO 021-G004 by Doyle et al. (2005), but its coordinates put it about $65^{\prime \prime}$ from the galaxy centre, beyond the optical 
size of the galaxy. Therefore, while the HI source may indeed be associated with this galaxy, we recommend caution with the cross-identification.

NGC 1365 The references in NED give redshifts closer to $1660 \mathrm{~km} \mathrm{~s}^{-1}$ which matches the velocity we measure for the narrow emission lines. As described above, the stellar absorption features (and also the broad emission lines) are offset from that and put the systemic velocity closer to $1530 \mathrm{~km} \mathrm{~s}^{-1}$

NGC 5037 The value adopted by NED is based on an HI measurement from Pisano et al. (2011). This is likely a misidentification, since the coordinates of the galaxy do not match those listed for it in their Table 2 . Instead, the velocity of $1904 \mathrm{~km} \mathrm{~s}^{-1}$ given in De Vaucouleurs et al. (1991) and the $1890 \mathrm{~km} \mathrm{~s}^{-1}$ reported by Mendel et al. (2008) are consistent with the velocity we find.

IC $4653 \mathrm{~A}$ redshift of $1890 \mathrm{kms}^{-1}$ is given in De Vaucouleurs et al. (1991), but a more recent measurement based on the Mg II feature was reported by Wegner et al. (2003). Its value of $1551 \mathrm{~km} \mathrm{~s}^{-1}$ is consistent with our measurement.

\subsection{Continuum Fitting and Subtraction}

Many of the emission lines we measure are superimposed on stellar absorption features, which need to be removed. Unlike Burtscher et al. (2020), whose purpose was to use these to derive the properties of the stellar population using a full spectral synthesis, our sole aim is to remove the stellar features. In addition, while stellar population models are currently only available at lower resolution, we are able to adopt a simpler approach using a theoretical library of individual stellar spectra from Coelho (2014) covering a range of temperature and surface gravity. The two advantages are that the high resolution of the library enables us to retain the full resolution of the spectra; and we fit $\sim 500 \AA$ segments independently in order to avoid difficulties arising from systematic effects due to a very long wavelength baseline. In order to maintain sufficient flexibility to account for the absorption, but to avoid over-subtraction of features that in some spectral types are very deep, we limited the range of template parameters to temperatures $4000 \leq T_{\text {eff }}(K) \leq 7000$ and surface gravities $2.5 \leq \log g\left(\mathrm{~cm} \mathrm{~s}^{-2}\right) \leq 4$, with solar abundances. The fitting was done using the Penalised Pixel Fitting routine pPXF (Cappellari \& Emsellem 2004; Cappellari 2017).

\subsection{Broad line region}

Table 1 shows that three of our targets (MCG-05-23-016, NGC 2992, and NGC 1365) are type 1.8-1.9, and therefore have measurable broad line emission at $\mathrm{H} \alpha$ and one case also $\mathrm{H} \beta$. Detailed fits to these and other HI lines have been performed by Schnorr-Müller et al. (2016a) in order to derive the extinction to the broad line region (BLR) as well as constrain the excitation. Here, we use a single Moffat function to represent the BLR, and fitted it simultaneously with the narrow lines. The example of NGC 1365, discussed already in Sec. 2.3, is shown in Fig. 1; the other two objects are more straightforward to fit and show no broad $\mathrm{H} \beta$. The BLR properties we find are consistent with those reported by Schnorr-Müller et al. (2016a).

\section{INTERSTELLAR MEDIUM VS OUTFLOW}

In this section we discuss whether the emission line profiles, shown in Fig. 2, are dominated by the interstellar medium (ISM) or the outflow. When referring to the ISM, we mean the ambient gas in the circumnuclear disk of the host galaxy. When referring to the outflow, we mean any gas that is not just photoionized by the AGN, but also kinematically disturbed by it. Spatially resolved studies (Fischer et al. 2017, 2018) have shown that there may be threshold radii, with gas closest to the AGN being driven out, beyond this a region where gas is still kinematically disturbed by the AGN, and finally a region where gas is photoionized by the AGN but remains undisturbed kinematically. Fischer et al. (2018) reported that for their sample of AGN, kinematically disturbed gas is seen out to $\sim 1.1 \mathrm{kpc}$. Our line profiles are integrated over a $1.8^{\prime \prime}$ box which corresponds to a much smaller radius of $\sim 150 \mathrm{pc}$. Even though, based on $\mathrm{L}_{[O I I I]}$, the AGN in our sample are an order of magnitude less luminous, we would still expect that at these radii the kinematics should be driven by the AGN rather than the host galaxy. In the following, we examine whether the excitation and kinematical properties of the AGN emission lines, in comparison to the matched inactive galaxies, support this asssumption. To do so, we quantify the line kinematics with two properties which are measured relative to the systemic velocity defined by stellar absorption features:

$v_{\text {peak }}$ is the velocity offset from systemic of the peak of the emission line. It has the same role as $v_{\text {int }}$ (line centroid) used by Bae \& Woo (2016) in their analysis of line profiles in biconical outflows. We use it to determine whether or not the core of the line is tracing the ambient ISM or the outflow. $v_{98}$ is the velocity above (or below) which one finds $98 \%$ of the line flux. We compare the absolute values of these and use whichever is the larger (we do not distinguish between the red and blue wings, and so $v_{98}$ as used here does not have a sign). This is motivated by the need to reliably estimate the maximum outflow velocity. It is similar in concept to other commonly used metrics (Rupke \& Veilleux 2011; Liu et al. 2013; Veilleux et al. 2013), and is an appropriate measure for the complex line profiles encountered here.

We have derived these for [SII], rather than the standard outflow tracer [OIII], because it is well detected in both active and inactive galaxies, allowing a comparison of these subsamples. In order to assess the level of bias in using [SII], Fig. 3 compares $v_{\text {peak }}$ and $v_{98}$ for the two lines in the AGN. With the exception of NGC 2110, there is good agreement between them, the most notable difference being that $v_{98}$ for the [OIII] line is about $25 \%$ larger (see also a comparison of the line profiles in Fig. 2).

\subsection{Excitation}

The left panel of Fig. 4 shows the standard $[\mathrm{NII}] / \mathrm{H} \alpha$ versus $[\mathrm{OIII}] / \mathrm{H} \beta$ ratios for our sample (see also Burtscher et al. 2020). For this plot, we used the flux integrated over the 

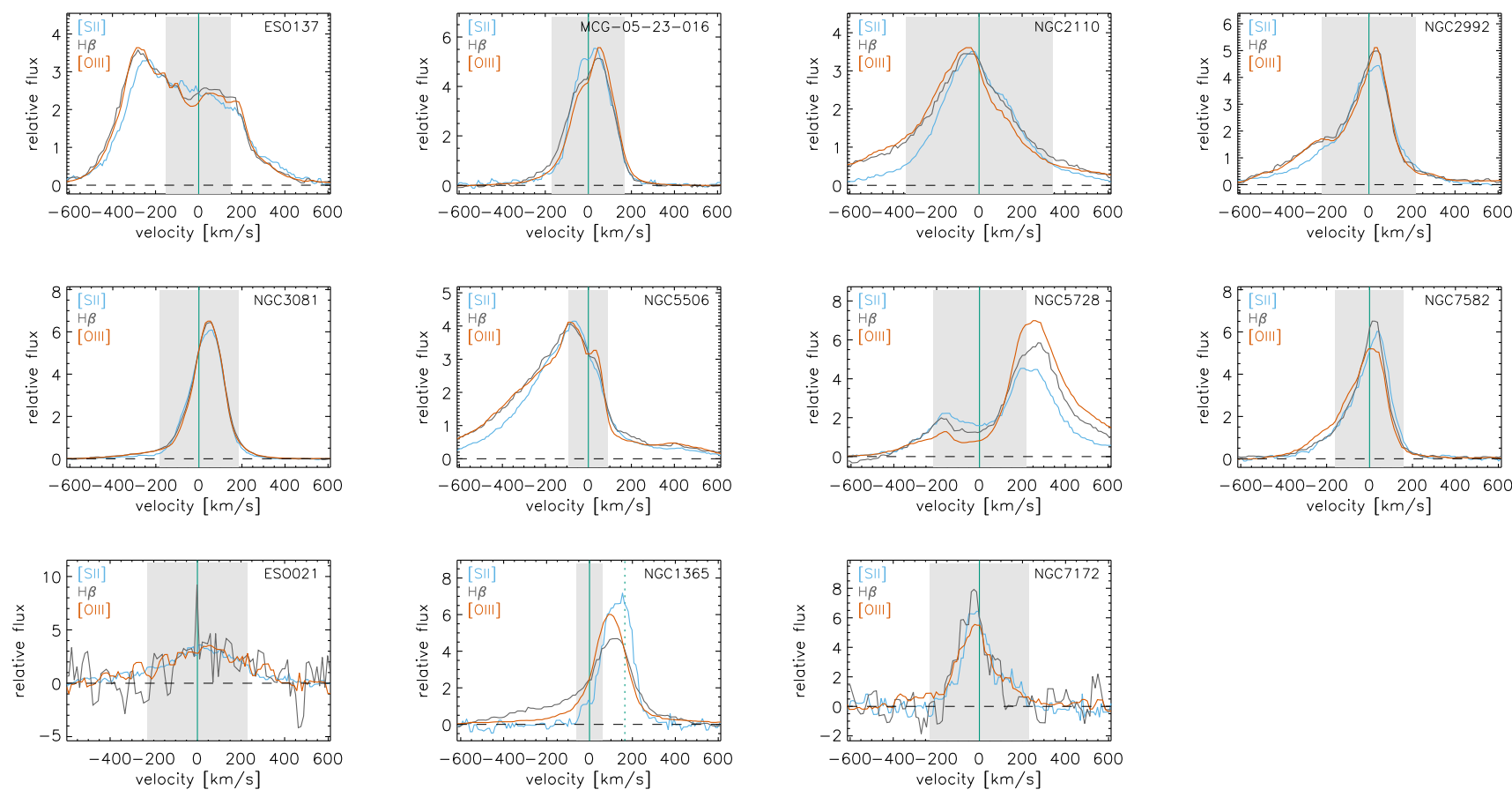

Figure 2. Comparison of central part of the line profiles of the AGN as a function of velocity, scaled relatively to the same flux within $\pm 250 \mathrm{~km} \mathrm{~s}^{-1}$. The $\mathrm{H} \beta$, [OIII], and [SII] profiles are shown in grey, red, and blue respectively. For visualisation purposes, the [SII] profile is a combination of the red side of the $6716 \AA$ line and the blue side of the $6731 \AA$ line, scaled to match where they overlap. These plots are of the data only, and not the fits. For reference, the FWHM of the stellar absorption profile is indicated by the shaded grey region. It is clear from this plot that the profiles show a variety of shapes that are generally inconsistent with the 'systemic + outflow' decomposition often adopted. We argue that the whole profile is dominated by, and hence traces, outflow. For reference, similar plots of the line profiles for the inactive galaxies are shown in Fig. A6 of the Appendix.
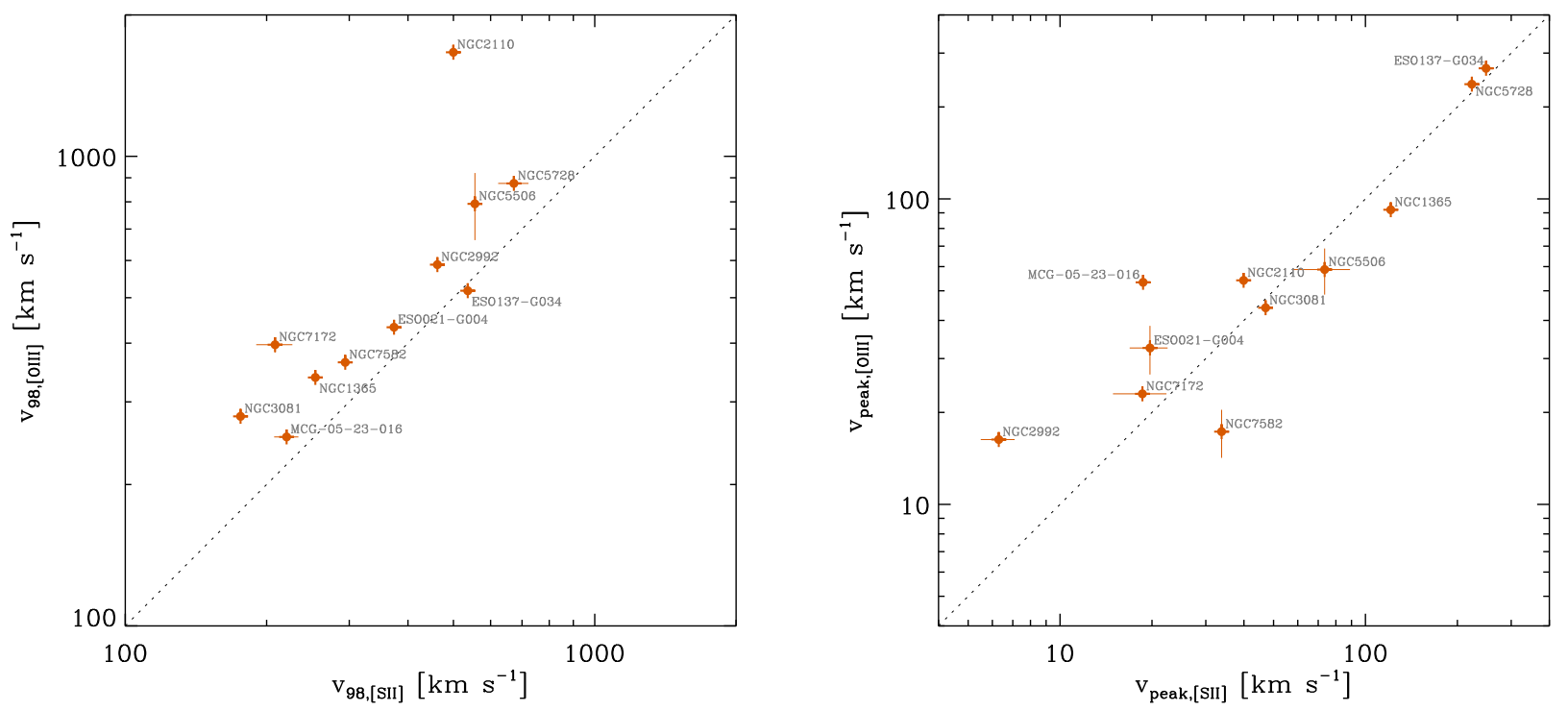

Figure 3. Comparison of the [SII] and [OIII] emission lines for the AGN, in terms of peak offset $v_{\text {peak }}$ from systemic (left) and outflow velocity $v_{98}$ (right). The dotted lines indicating a 1:1 correspondance show that in each case the two lines are very similar. These show that there is little difference between the kinematics traced by these two emission lines. The left panel reflects the well-known result that [OIII] has broader wings than [SII] (Veilleux 1991); in this case the velocity difference is about 25\%. Only NGC 2110 is an outlier, and inspection of Fig. 2 shows that its [OIII] line does have very broad wings. In the right panel, the peak velocity offsets are well correlated to $10-20 \mathrm{~km} \mathrm{~s}^{-1}$ which is the expected level of systematics. 

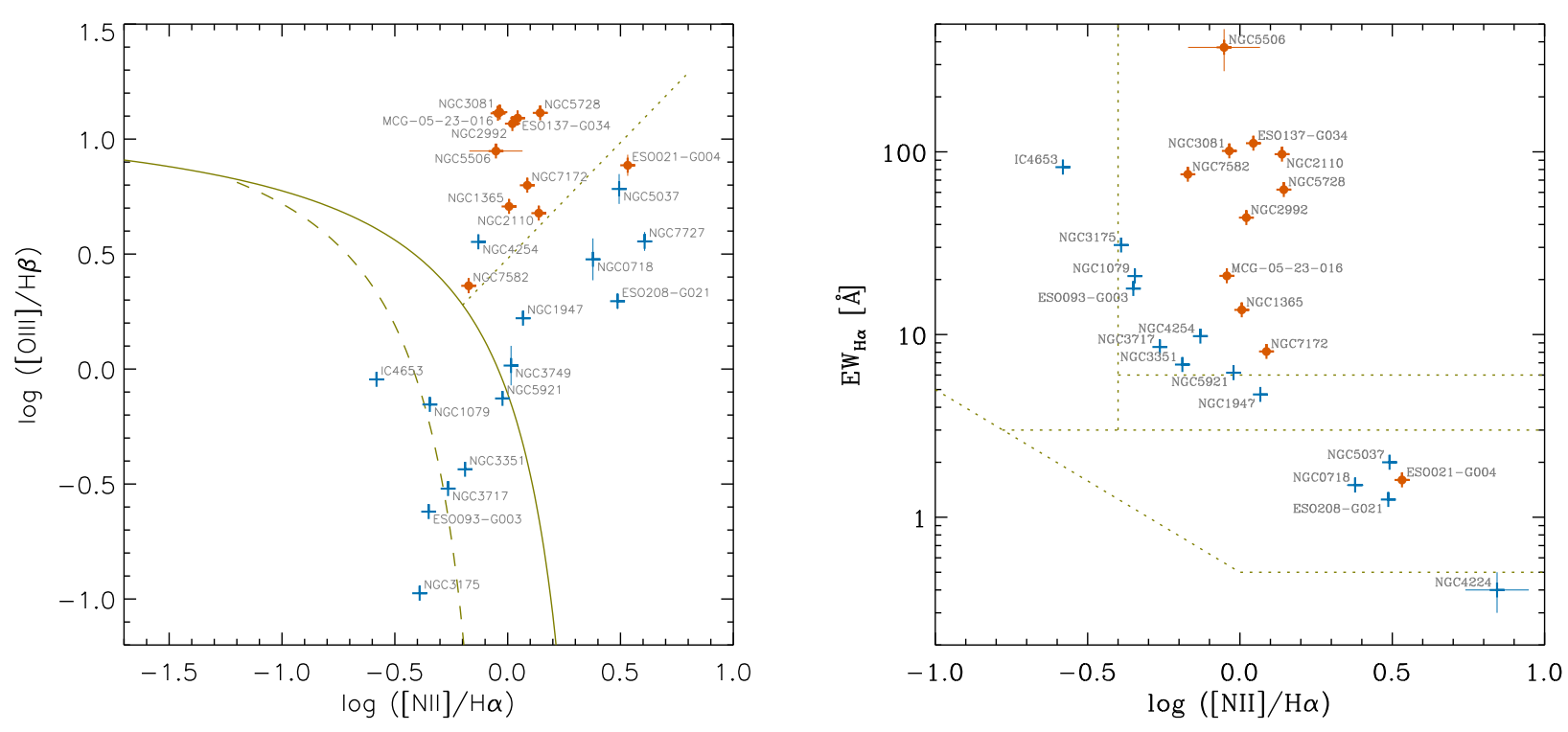

Figure 4. Comparison of measured emission line quantites for AGN (red with filled circles) and inactive galaxies (blue). Left: standard $[\mathrm{NII}] / \mathrm{H} \boldsymbol{\alpha}$ versus $[\mathrm{OIII}] / \mathrm{H} \beta$ diagnostic ratios, with the Kewley et al. (2001) extreme starburst line (solid) and Kauffmann et al. (2003) classification line (dashed), as well as the Cid Fernandes et al. (2010) Seyfert/LINER coarse separation (dotted). Among the inactive galaxies, NGC 4224 is omitted because we could not measure the $\mathrm{H} \beta$ line flux. Right: The WHAN plot of the equivalent width of H $\alpha$ versus the $[\mathrm{NII}] / \mathrm{H} \alpha$ ratio. The dotted lines are shown for reference to Figs. 1 and 6 of Cid Fernandes et al. (2011). The key point here is that the AGN and inactive galaxies form distinct sequences. These panels show that the emission lines in the AGN sample are dominated by the AGN photoionization.

full line profile, and so it simply shows that the whole of the line emission is dominated by AGN photoionization rather than other processes. Although some AGN lie close to the border with the LINER region, it is known that there is considerable overlap between Seyferts and LINERs at that boundary. The line equivalent width should be considered a third dimension of the standard line ratio plot because it shows that, at high $[\mathrm{NII}] / \mathrm{H} \alpha$ ratios, AGN photoionization is generally associated with much brighter line emission than would be expected from (post-AGB) stellar photoionization (Stasińska et al. 2008). Cid Fernandes et al. (2010, 2011) proposed its use in an alternative and complementary 'WHAN' diagnostic plot of the $\mathrm{H} \alpha$ equivalent width versus the $[\mathrm{NII}] / \mathrm{H} \alpha$ ratio. This is shown for our sample in the right panel of Fig. 4, where the AGN and inactive galaxies form distinct sequences. The location of the AGN in these two plots confirms that their emission lines are indeed AGN dominated. The only exception is ESO 021-G004, which has a remarkably low $\mathrm{H} \alpha$ equivalent width. This is surprising because the sample selection ensures that all the AGN are of similar moderate luminosity, and Table 6 shows that ESO 021-G004 is unremarkable in this respect. Instead, our estimate of the extinction to the narrow lines based on the $\mathrm{H} \alpha / \mathrm{H} \beta$ ratio given in Table 4 indicates that this object (together with NGC 7172) has $A_{V}>3$ mag and hence its intrinsic line luminosity is a factor 15 higher than that observed.

The inactive galaxies occupy a rather different locus on these plots, following a relatively narrow track that extends from pure star-forming galaxies to LINERs photoionized by post-AGB stars. This sequence clarifies that NGC 4254, while it is formally among the Seyferts in the left panel of
Fig. 4 (bearing in mind that there is overlap with LINERs across that boundary), does indeed fit better among the inactive galaxies. These objects were selected to be matched to the AGN host galaxies (Davies et al. 2015; Rosario et al. 2018) and so are similar on large scales. An analysis of the stellar population in the central $\sim 300 \mathrm{pc}$ shows that they are also similar on small scales (Burtscher et al. 2020). These studies imply that if the AGN were to become inactive, we would expect them to look like the inactive sample, with similar photoionization properties and line strengths. This difference is apparent also in the kinematics, as discussed next.

\section{$3.2 \quad$ Velocities}

Fig. 5 plots the distributions of the maximum velocity $v_{98}$ and the velocity offset $v_{\text {peak }}$ of the emission line peak from the systemic velocity. The maximum velocity is rather similar for the active and inactive galaxies. The reason is that for the inactive galaxies, $v_{98}$ traces the edge of an outflow wing in the line profile that is distinct from the dominant systemic component. In contrast, for the active galaxies, the outflow is the dominant part of the line profile, and so $v_{98}$ traces the edge of the bulk of the line emission. This is clarified by the distribution for the peaks of the emission lines, with $v_{\text {peak }}$ for the AGN being significantly offset from the systemic velocity. This is not the case for inactive galaxies, for which, as can be seen in Fig. 5, the median absolute offset of the emission line peak from systemic is $11 \mathrm{~km} \mathrm{~s}^{-1}$. Such small values are consistent with irregularities in a distribution that basically traces host galaxy rotation. It strongly suggests that the line peak for inactive galaxies is tracing 

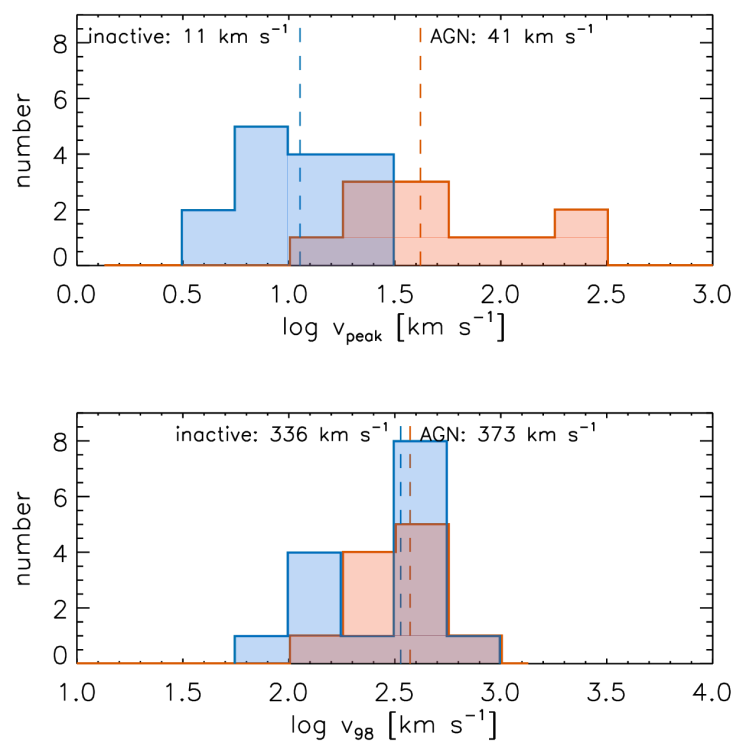

Figure 5. Comparison of kinematic emission line properties for AGN (red) and inactive galaxies (blue), with median values indicated by the vertical dashed lines. Upper: velocity offset $v_{\text {peak },[S I I]}$ of the peak of the [SII] emission line from systemic (as measured by stellar absorption features). Lower: maximum velocity $v_{98,[S I I]}$ of the $[\mathrm{SII}]$ line from systemic. Both quantities are given as absolute values. These show that $v_{98}$ is similar for the active and inactive galaxies, while $v_{\text {peak }}$ distinguishes between them rather well. This is because the high value of $v_{98}$ for the inactive galaxies is due to an outflow wing on the line profile that is distinct from the systemic component; while for the AGN the outflow is the dominant part and so affects $v_{\text {peak }}$ as well.

the ambient ISM, as expected. In contrast, the offsets for all the AGN are larger than the median of the inactive galaxies; and for $2 / 3$ of the AGN they are greater than the maximum $28 \mathrm{~km} \mathrm{~s}^{-1}$ for the inactive galaxies. The median of $42 \mathrm{~km} \mathrm{~s}^{-1}$ for the AGN is a significant offset for the line peak and, if associated with host galaxy rotation, would imply a highly asymmetric (one-sided) line distribution. Instead, we conclude that in the central few hundred parsecs of AGN, even the peak of the emission lines is tracing outflow.

\subsection{Outflowing versus ambient gas}

Some AGN, typified by NGC 5728 and ESO 137-G034, not only have high maximum outflow speeds, but more dramatically the peak of the emission line is offset from systemic by $>30 \mathrm{~km} \mathrm{~s}^{-1}$. These tend to be those with stronger emission lines, and are clearly the innermost regime noted by Fischer et al. (2018), where the line emission originates from gas being driven out by the AGN. The line profiles of these objects are typified by a wide double-peaked profile either side of systemic, which can be understood in terms of the approaching and receding sides of an outflow. In these objects any systemic contribution to the line is negligible. A library of line profiles from biconical outflows has been modelled by Bae \& Woo (2016), covering a variety of orientations, opening angles, and differential extinction. Although at lower res- olution than our spectra, they show clearly that, in addition to complex profiles such as those discussed above, one can expect some outflows to have rather narrow unremarkable profiles because of their more edge-on orientation. This is particularly important for Seyfert $2 \mathrm{~s}$ which are by definition closer to edge-on. We have reproduced some examples at a higher resolution in Fig. 6. These cover inclinations from 40$80^{\circ}$ and have the emission from behind a host galaxy disk blocked by varying amounts. They qualitatively match the profiles we observe, for example the orange profile in the left panel $\left(i_{\text {inc }}=40^{\circ}\right.$, with half of the emission behind the disk blocked) is similar to ESO 137-G034, while the green profile in the right panel $\left(i_{\text {inc }}=80^{\circ}\right.$, with all of the emission behind the disk blocked) is more like NGC 7582. Generally, these model profiles include not only double-peaked profiles, but also narrower profiles, both with and without prominent wings. This helps understand some of the less remarkable profiles such as those for MCG-05-23-016 or NGC 3081, both of which clearly show some characteristics of outflow. In particular, MCG-05-23-016 shows a distinct break at systemic typical of a double-peak from a more inclined bicone, while for NGC 3081 the profile is smoother but its peak is far offset from systemic suggesting that the approaching side may be obscured behind the galaxy disk. The most curious case is NGC 1365. Sec. 2.3 and Fig. 1 demonstrate that the narrow line emission is offset from the systemic velocity (and also the broad lines) by $165 \mathrm{~km} \mathrm{~s}^{-1}$, and is instead associated with a neutral outflow traced by the NaI D lines. Additional evidence of a prominent outflow comes from larger scale data and, for a bicone model, Hjelm \& Lindblad (1996) derived an inclination of $35^{\circ}$ to the line of sight and a half opening angle of $50^{\circ}$. Similarly, the velocity map of [OIII] presented by Venturi et al. (2018) also shows projected velocities reaching to $\sim 150 \mathrm{~km} \mathrm{~s}^{-1}$ (although not more) within the central arcminute. Thus, as our data also show, it is reasonable to expect that the outflow should dominate the line emission in the much smaller $1.8^{\prime \prime}$ aperture.

As a final check about whether there is a measurable systemic component, we have examined the [SII] doublet ratio, and also the $[\mathrm{OIII}] / \mathrm{H} \beta$ ratio (which directly affects the derived density for the $\log U$ method) as a function of velocity. There are clearly trends with velocity (similar to those reported by Veilleux 1991), especially between the redshifted and blue-shifted emission, reflecting difference in the approaching and receeding sides of the outflow. However, even at the spectral resolution and signal-to-noise of these data, there is no evidence for changes in either ratio associated with the systemic velocity. Together with the assessment of the profile shape above, this suggests that integrating over the full line profiles will not lead to any bias in the resulting density due to a (sub-dominant) systemic component in the line profile.

Our conclusion in this section is that, while a 'systemic + outflow' decomposition can be appropriate in many cases, it fails for the nuclear observations presented here. Instead the observed emission lines are fully dominated by an AGN photoionized outflow. The reasons include (i) that our spectra are extracted from small apertures that trace gas within $\sim 150$ pc from moderately luminous AGN, (ii) our ability to make an independent measure of the systemic velocities from the stellar continuum, (iii) the complexity of the line profiles in Fig. 2 which either have a dip at the systemic veloc- 

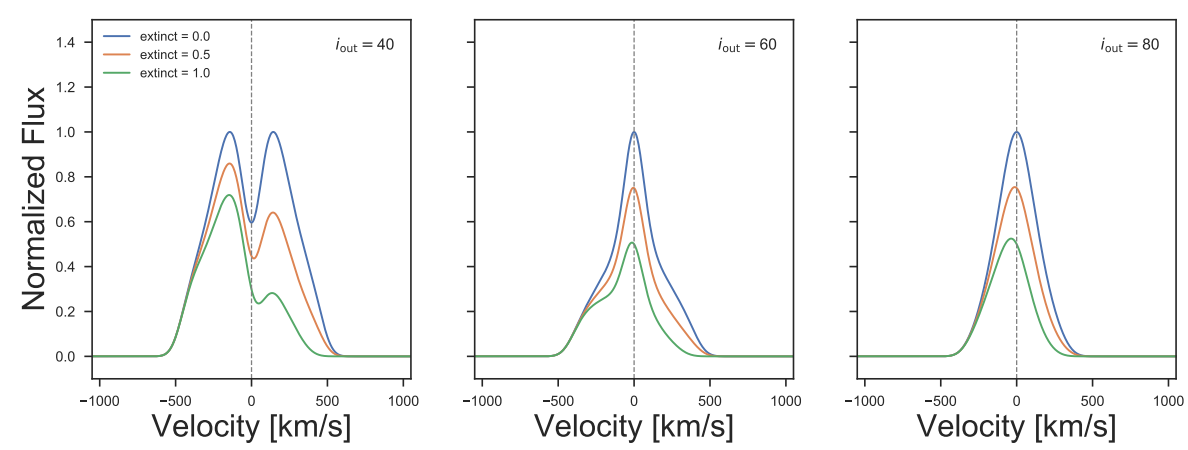

Figure 6. Examples of the variety of line profiles that can be produced by a biconical outflow, depending on orientation (panels are for $i_{\text {out }}=40^{\circ}, 60^{\circ}, 80^{\circ}$ to the line of sight) and dust obscuration (colours denote a fraction extinct $=0,0.5,1$ of the emission from behind the host galaxy disk is blocked). These particular examples are for a bicone with inner and outer half-opening angles of $20^{\circ}$ and $40^{\circ}$, with a velocity profile that accelerates to $500 \mathrm{~km} \mathrm{~s}^{-1}$ at a turnover radius and then decelerates. A host galaxy disk oriented at $60^{\circ}$ provides obscuration. This can affect not just the rear cone, but also produce more complex effects in the line proifile if part of each cone is obscured. A full library at lower velocity resolution is presented by Bae \& Woo (2016). The examples here include double-peaked profiles, as well as rather narrower profiles, both with and without prominent wings. They demonstrate that even the narrower profiles among our AGN are consistent with an outflow origin.

ity or continue smoothly across it, and (iv) that we know it is easy to find reasonable parameter sets for biconical outflow models that reproduce both the dramatic and the more unremarkable profiles. Our interpretation is that in every case, the whole profile is probing outflowing gas. It is difficult to estimate how much a systemic (stationary) component might contribute to the line. Based on comparison of the equivalent widths of the lines in the active and inactive galaxies, we would estimate that a systemic component contributes $<10 \%$ to the total line flux. As such, for the analysis of the outflow density in Sec. 4 we use the full integrated line flux.

\section{DENSITY AND MASS MEASUREMENTS}

In this Section we explore three independent ways to measure the density of the ionized gas. These include the most commonly used method based on the [SII] doublet ratio, an alternative proposed by Holt et al. (2011) that uses a combination of [SII] and [OII] line ratios, and a method recently introduced by Baron \& Netzer (2019) that is based on the ionization parameter. We indicate their main limitations and merits, and calculate the density ranges for our sample using each method. We compare these ranges, and use photoionization models to understand the differences between them and the impact on the implied ionized gas mass.

\section{1 [SII] doublet ratio method}

The most commonly used electron density tracer (here referred to as the doublet method) uses the [SII] $\lambda 6716,6731 \AA$ doublet, because it only requires a measurement of the ratio of two strong emission lines in a convenient and clean part of the optical spectrum, and the physics of the excitation and de-excitation means that density - covering a range commonly found in HII regions - dominates the emitted line ratio (Osterbrock 1989). An additional advantage is that, because the lines are necessarily close in wavelength, the derived density is unaffected by extinction.
There are, however, situations where this ratio can give misleading results. Because the two lines are separated by only $14.4 \AA$, corresponding to $650 \mathrm{~km} \mathrm{~s}^{-1}$, deblending the doublet can become unreliable at moderate spectral resolution. This is particularly important for complex line profiles. As an example, in a detailed study of the ionized and molecular gas in the circumnuclear region of NGC 5728, Shimizu et al. (2019) compared several methods of measuring the electron density using both high and moderate resolution data. They showed that for data with $\mathrm{R}<4000$, blending of the [SII] line profiles in this object leads to an increasing discrepancy in the derived density at smaller radii - with an order of magnitude under-estimation at radial scales below 500 pc. For our sample, Figs. A1 and A3 show that the high spectral resolution and signal-to-noise of our data mean that the line profiles of both active and inactive galaxies can be robustly determined.

It is well known that [SII] cannot probe high densities because collisional de-excitation dominates above $10^{4} \mathrm{~cm}^{-3}$ where the ratio saturates at $\sim 0.45$, its asymptotic value. We note that this effect should not bias our measurements because none of the electron densities derived from the [SII] doublet in our sample exceed $1000 \mathrm{~cm}^{-3}$.

A less well known bias can arise from the impact of the stellar continuum when the equivalent width of the [SII] lines is low. This is illustrated in Fig. 7 for NGC 7727. The apparent line ratio of 0.98 (left panel) is rather less than the actual line ratio of 1.21 (centre panel) due to the stellar absorption feature under the $6716 \AA$ line. It seems likely that this is dominated by Fe I. Since the feature is weak, it only affects lines with equivalent width $\lesssim 10 \AA$. But the impact on the resulting derived densities can be significant. For the inactive galaxies in our sample, for which the median equivalent width is $1.5 \AA$, the right panel of Fig. 7 shows that failing to correct for this effect leads to a factor 2.5 over-estimation of the typical density. In contrast, for the stronger lines in the AGN the bias is negligible.

An additional caveat is that this method yields the electron density in the region where the line is emitted. In AGN, the emission from low ionization transitions such as 

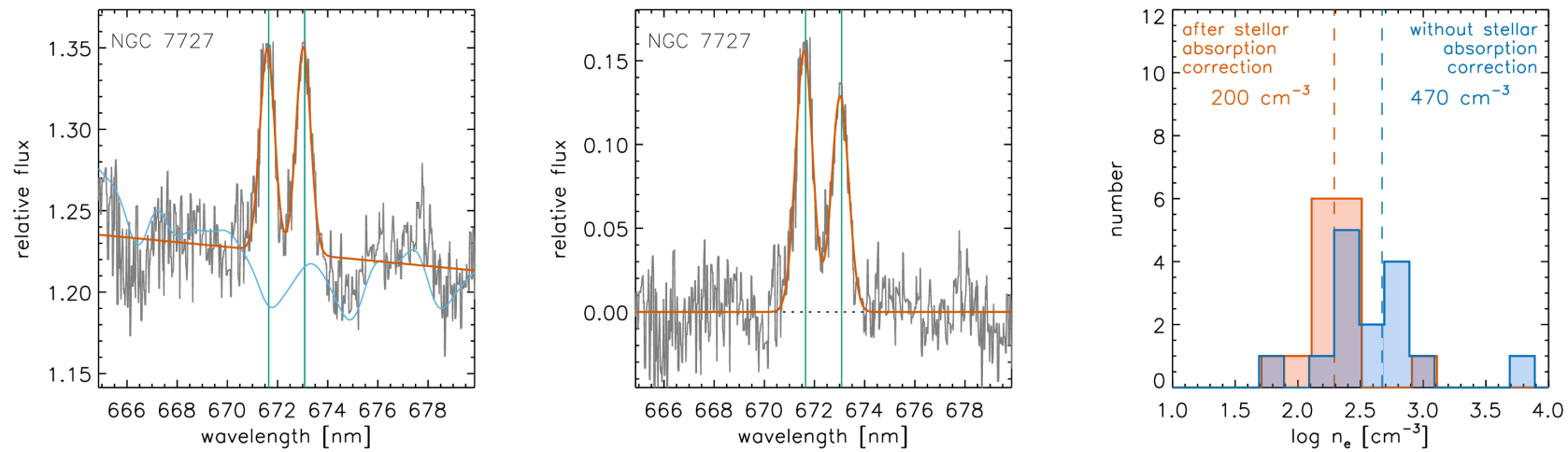

Figure 7. Bias in density measurement for a weak [SII] doublet. Left: the observed spectrum (grey line) for NGC 7727 overplotted with the stellar continuum (blue line) and a fit to the emission line doublet approximating the stellar continuum with a linear function (red line). The absorption feature under the $6716 \AA$ line is likely to be dominated by Fe I. The vertical green lines indicate the line centres for the doublet. Centre: as for the left panel but after subtracting the fitted stellar continuum. Right: the derived densities for all the inactive galaxies without (blue) and after (red) correction for the stellar continuum.

Table 2. Measurements of [SII] doublet, and derived densities, for active and inactive galaxies.

\begin{tabular}{lccccc}
\hline Object & $v_{98,[S I I]}\left(\mathrm{km} \mathrm{s}^{-1}\right)$ & $v_{\text {peak, }[\text { SII }]}\left(\mathrm{km} \mathrm{s}^{-1}\right)$ & $\mathrm{EW}_{[S I I]}(\AA)$ & {$[\mathrm{SII}]$ doublet ratio } & $\log n_{e}\left(\mathrm{~cm}^{-3}\right)$ \\
\hline ESO 137-G034 & $536 \pm 3$ & $248.3 \pm 0.8$ & $39.6 \pm 0.3$ & $0.96 \pm 0.01$ & $2.76 \pm 0.01$ \\
MCG-05-23-016 & $221 \pm 13$ & $18.7 \pm 0.4$ & $4.9 \pm 0.1$ & $0.98 \pm 0.01$ & $2.74 \pm 0.01$ \\
NGC 2110 & $499 \pm 7$ & $41.5 \pm 0.8$ & $51.4 \pm 0.7$ & $1.09 \pm 0.02$ & $2.54 \pm 0.03$ \\
NGC 2992 & $461 \pm 4$ & $13.9 \pm 1.1$ & $16.3 \pm 0.3$ & $1.02 \pm 0.02$ & $2.66 \pm 0.02$ \\
NGC 3081 & $176 \pm 2$ & $46.6 \pm 1.4$ & $23.4 \pm 0.6$ & $0.99 \pm 0.03$ & $2.72 \pm 0.05$ \\
NGC 5506 & $554 \pm 10$ & $73.5 \pm 15.7$ & $111.8 \pm 3.3$ & $1.17 \pm 0.04$ & $2.37 \pm 0.08$ \\
NGC 5728 & $679 \pm 18$ & $227.6 \pm 1.2$ & $18.9 \pm 0.5$ & $1.11 \pm 0.07$ & $2.76 \pm 0.03$ \\
NGC 7582 & $294 \pm 6$ & $33.8 \pm 1.0$ & $9.5 \pm 0.2$ & $0.96 \pm 0.03$ & $2.77 \pm 0.04$ \\
ESO 021-G004 & $374 \pm 6$ & $19.7 \pm 2.8$ & $2.0 \pm 0.2$ & $1.22 \pm 0.03$ & $2.26 \pm 0.07$ \\
NGC 1365 & $254 \pm 2$ & $121.0 \pm 0.7$ & $1.6 \pm 0.1$ & $1.23 \pm 0.02$ & $2.24 \pm 0.06$ \\
NGC 7172 & $209 \pm 19$ & $18.6 \pm 3.6$ & $2.0 \pm 0.3$ & $1.25 \pm 0.19$ & $2.20 \pm 0.77$ \\
& & & & & \\
ESO 093-G003 & $140 \pm 5$ & $14.2 \pm 1.1$ & $3.4 \pm 0.1$ & $1.18 \pm 0.03$ & $2.36 \pm 0.08$ \\
ESO 208-G021 & $548 \pm 19$ & $11.3 \pm 2.4$ & $1.7 \pm 0.1$ & $1.17 \pm 0.07$ & $2.37 \pm 0.14$ \\
NGC 0718 & $470 \pm 128$ & $9.6 \pm 1.6$ & $0.8 \pm 0.1$ & $1.17 \pm 0.06$ & $2.41 \pm 0.13$ \\
NGC 1079 & $152 \pm 1$ & $6.2 \pm 0.6$ & $3.5 \pm 0.1$ & $1.27 \pm 0.02$ & $2.13 \pm 0.04$ \\
NGC 1947 & $537 \pm 44$ & $9.1 \pm 1.5$ & $4.1 \pm 0.3$ & $1.24 \pm 0.07$ & $2.20 \pm 0.19$ \\
NGC 3175 & $106 \pm 3$ & $8.9 \pm 0.4$ & $4.1 \pm 0.1$ & $1.24 \pm 0.05$ & $2.24 \pm 0.12$ \\
NGC 3351 & $285 \pm 33$ & $3.4 \pm 1.1$ & $1.2 \pm 0.1$ & $1.20 \pm 0.06$ & $2.33 \pm 0.15$ \\
NGC 3717 & $473 \pm 27$ & $28.4 \pm 1.2$ & $1.9 \pm 0.1$ & $1.23 \pm 0.03$ & $2.25 \pm 0.06$ \\
NGC 3749 & $410 \pm 31$ & $11.5 \pm 2.4$ & $0.9 \pm 0.1$ & $1.26 \pm 0.08$ & $2.17 \pm 0.24$ \\
NGC 4224 & $595 \pm 38$ & $16.0 \pm 9.0$ & $0.9 \pm 0.1$ & $0.84 \pm 0.04$ & $2.98 \pm 0.07$ \\
NGC 4254 & $144 \pm 11$ & $27.5 \pm 1.8$ & $1.0 \pm 0.2$ & $1.12 \pm 0.17$ & $2.49 \pm 0.34$ \\
NGC 5037 & $337 \pm 5$ & $28.0 \pm 2.4$ & $1.8 \pm 0.1$ & $1.31 \pm 0.03$ & $2.02 \pm 0.11$ \\
NGC 5921 & $392 \pm 13$ & $6.6 \pm 0.6$ & $1.9 \pm 0.1$ & $1.16 \pm 0.03$ & $2.39 \pm 0.06$ \\
NGC 7727 & $332 \pm 6$ & $25.1 \pm 3.0$ & $1.1 \pm 0.3$ & $1.21 \pm 0.04$ & $2.29 \pm 0.09$ \\
IC 4653 & $69 \pm 1$ & $3.4 \pm 0.4$ & $12.9 \pm 0.1$ & $1.37 \pm 0.01$ & $1.72 \pm 0.05$ \\
\hline
\end{tabular}

${ }^{a} \mathrm{EW}_{[S I I]}$ is for the $6716 \AA$ line in the doublet.

[SII], [NII], and [OI] is enhanced (compared to HII regions around stars) by an extended partially ionized zone (Osterbrock 1989). In such regions of a cloud, where the gas is mostly neutral, the electron density will not necessarily be comparable to the hydrogen gas density. In some circumstances this can become a critical issue and we return to it in Sec. 4.4.

We have measured the $[\mathrm{SII}]$ doublet lines in the inactive galaxies after subtracting the stellar continuum, which was fitted using a combination of high resolution models as described in Sec. 2.4. The lines were fitted using Gaus- sian components: two for each line in the inactive galaxies, while the active galaxies required more typically three components (and for NGC 5728 we used four components). As a constraint to the fit, we required the profiles of the two lines in the doublet to be the same, and hence we are measuring a single ratio for the full line emission. We used the routine mpfitfun (Markwardt 2009); and we propagated the uncertainties of the parameters provided by this routine to the relevant properties using Monte-Carlo techniques. The profile fits are shown in Fig. A3 for the inactive galaxies and Fig. A1 for the active galaxies. The measured proper- 


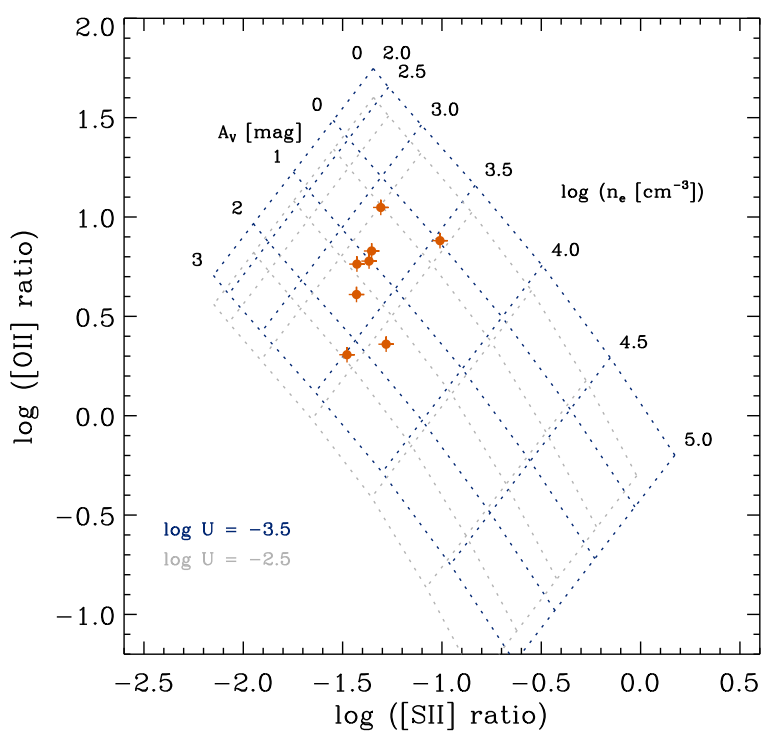

Figure 8. Location of the measured [OII] and [SII] ratios for the AGN in our sample, in comparison to photoionization models that trace a grid of extinction $A_{V}$ versus electron density $n_{e}$. Models are shown for solar metallicity and a standard AGN spectral energy distribution; but for two different ionization parameters covering the range found for the AGN here.

ties of the [SII] doublet lines are summarised in Table 2 for the complete active and inactive sample. The median density for the inactive galaxies is $200 \mathrm{~cm}^{-3}$, while that for the active galaxies is $460 \mathrm{~cm}^{-3}$.

In terms of density and outflow velocity, NGC 4224 has characteristics more like an AGN outflow than the inactive galaxy that it is. It is excluded from the left panel of Fig. 4 because we could not measure the $\mathrm{H} \beta$ flux. However, its ratio $\log [N I I] / H \alpha \sim 0.85$ would put it well into the LINER region. We have found no evidence that this object might be an AGN, and indeed it has a very low $\mathrm{H} \alpha$ equivalent width of $<0.5 \AA$. Although it is a spiral galaxy classified as Sa (Davies et al. 2015), it is one of the few inactive galaxies in our sample for which the central optical spectrum shows no indication of any stellar population younger than $\sim 3 \mathrm{Gyr}$ (Burtscher et al. 2020). And despite being a member of the Virgo Cluster (Binggeli et al. 1985), the outer parts of the galaxy show no signs of being disturbed (Buta et al. 2015). The line characteristics indicate that there is an outflow, and we speculate that it could be either a fossil AGN outflow be driven by post-AGB stars.

\subsection{Auroral and Transauroral line method}

To avoid the limitations of the [SII] doublet ratio, Holt et al. (2011) proposed an alternative method that has now been applied to a variety of different types of objects, and been shown to be sensitive to higher densities (Holt et al. 2011; Rose et al. 2018; Santoro et al. 2018; Shimizu et al. 2019). We will refer to this as the TA method because it uses the transauroral lines [SII] $\lambda 4069,4076$ and the auroral lines [OII] $\lambda 7320,7331$ (each of these is itself

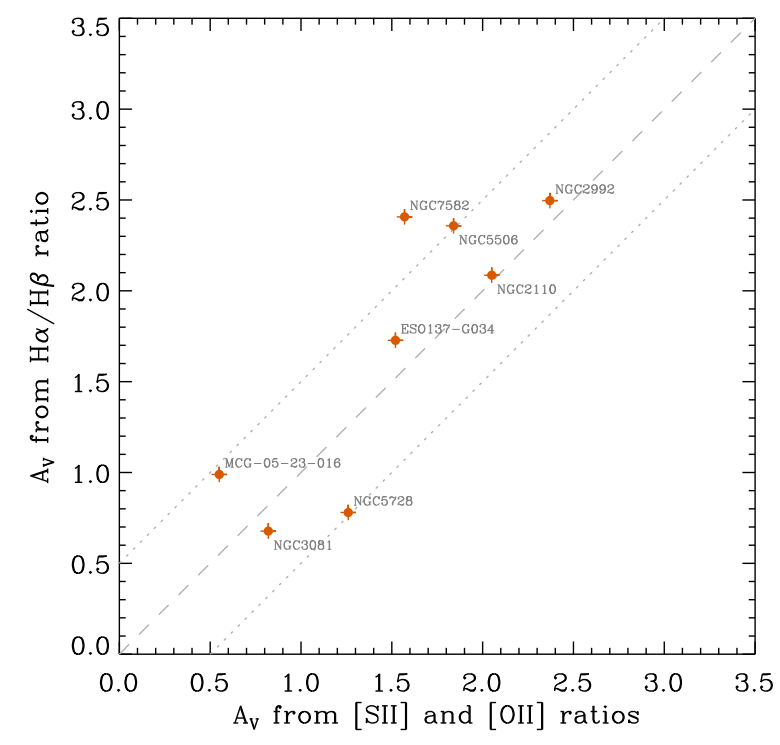

Figure 9. Comparison of extinction $\mathrm{A}_{V}$ derived from the [SII] and $[\mathrm{OII}]$ line ratios to that derived from the $\boldsymbol{H \alpha} / \boldsymbol{H} \boldsymbol{\beta}$ ratio. The dashed line indicates a 1:1 ratio and the dotted lines a $\pm 0.5 \mathrm{mag}$ range.

a doublet but their separations of $\sim 1 \AA$ are sufficiently small that at the spectral resolution here they can be considered single lines) which have higher critical densities. These are used together with the stronger lines to give the ratios $[\mathrm{SII}] \lambda(4069+4076) /[\mathrm{SII}] \lambda(6716+6731)$ and [OII $] \lambda(3726+3729) /[\mathrm{OII}] \lambda(7320+7331)$.

The way these lines are used to estimate density differs fundamentally from the doublet method. Rather than providing a direct measure of $n_{e}$ in the gas where the line emission originates, the ratios of summed doublet fluxes are compared to those produced in photoionization models. The models take account internally of how $n_{e}$ (on which the emitted lines depend) varies through the cloud, and hence how the resulting cumulative line ratios are related to $n_{H}$. As such, the method traces $n_{H}$ rather than $n_{e}$ in a (constant density) cloud; but typically one then equates $n_{H}$ and $n_{e}$ as if the gas were fully ionized. The [OII] lines arise predominantly in the fully ionized gas, but they are far apart in wavelength and so the impact of extinction needs to be addressed. This is done using the [SII] lines, which originate from different regions of the cloud and span a shorter wavelength range, so that their ratio has a different dependency on density and extinction. The pair of ratios then provides a reference basis for photoionization model grids in which density and extinction are approximately orthogonal.

The primary advantages of the method are that it is sensitive to higher densities, which is now known to be important when measuring outflow rates; and it uses the summed flux in each doublet and thus is less sensitive to the details of the line profile. However, it too has some limitations. Most critically from the observational perspective, the auroral and transauroral lines are very weak. Figs. A4-A5 show that their flux is typically only about $10 \%$ of the strong [SII] and [OII] lines, and so signal-to-noise can be a major restriction. For 
Table 3. Measurements of the [SII] and [OII] line ratios used by the auroral/transauroral method, and derived densities and extinctions for active galaxies

\begin{tabular}{lcccc}
\hline Object & $\begin{array}{c}\log [\mathrm{SII}] \text { ratio } \\
(4069+4076) /(6716+6731)\end{array}$ & $\begin{array}{c}\log [\mathrm{OII}] \mathrm{ratio} \\
(3726+3729) /(7320+7331)\end{array}$ & $\log n_{e}\left(\mathrm{~cm}^{-3}\right)$ & $\mathrm{A}_{V}(\mathrm{mag})$ \\
\hline & & & & \\
ESO 137-G034 & $-1.43 \pm 0.01$ & $0.76 \pm 0.02$ & $3.13 \pm 0.02$ & $1.52 \pm 0.04$ \\
MCG-05-23-016 & $-1.01 \pm 0.02$ & $0.88 \pm 0.02$ & $3.46 \pm 0.02$ & $0.55 \pm 0.04$ \\
NGC 2110 & $-1.28 \pm 0.01$ & $0.36 \pm 0.01$ & $3.61 \pm 0.02$ & $2.05 \pm 0.03$ \\
NGC 2992 & $-1.48 \pm 0.02$ & $0.31 \pm 0.03$ & $3.43 \pm 0.03$ & $2.37 \pm 0.06$ \\
NGC 3081 & $-1.31 \pm 0.02$ & $1.05 \pm 0.01$ & $3.01 \pm 0.02$ & $0.82 \pm 0.04$ \\
NGC 5506 & $-1.43 \pm 0.03$ & $0.61 \pm 0.02$ & $3.27 \pm 0.03$ & $1.84 \pm 0.05$ \\
NGC 5728 & $-1.35 \pm 0.03$ & $0.83 \pm 0.02$ & $3.16 \pm 0.04$ & $1.26 \pm 0.06$ \\
NGC 7582 & $-1.36 \pm 0.04$ & $0.78 \pm 0.05$ & $3.28 \pm 0.05$ & $1.57 \pm 0.11$ \\
\hline
\end{tabular}

the same reason, the continuum level fitted around the lines can have a significant impact on the measured line flux and so subtracting the stellar continuum is mandatory.

The conversion of the measured ratios to a density is also not as straightforward as for the [SII] doublet ratio because it depends on photoionization models. Holt et al. (2011) assessed the impact of changes in the spectral index and ionization parameter, and argued that they are relatively unimportant. Our own calculations (described below) indicate that these should not be ignored: Fig. 8 shows that the ionization parameter can change the derived density by a factor 2-3; and changing the metallicity has a comparable impact. In addition, the wide wavelength range required to cover all the lines means that the effects of extinction must be included when fitting the models to the data. And the choice of extinction model will also have an impact on density derived from the line ratios.

For the AGN in which all the necessary lines can be measured, we have fitted the [SII] and [OII] doublets in a similar way to that described previously in Sec. 4.1. Because the [SII] $\lambda 6716,6731$ lines are the strongest, we have fitted those first in order to determine the centerings and FWHMs of the Gaussian components in the line profile. These were then fixed when fitting the remaining three doublets, allowing only the scalings to vary in such a way that the profiles of the two lines within each doublet match, while allowing flexibility in terms of differences between the doublets. The resulting profile fits for each doublet are shown in Figs. A4-A5. As before we propagated the uncertainties in fitted parameters to the summed fluxes and hence ratios using Monte-Carlo techniques.

To estimate densities from the line ratios, we have performed calculations using CLOUDY v17 (Ferland et al. 2017). We calculated a grid of photoionization models covering a large range in ionization parameter and hydrogen density, with details and assumptions similar to those presented in Appendix A of Baron \& Netzer (2019). We adopted a standard AGN spectral energy distribution (SED) with a mean energy of an ionizing photon of 2.56 Ryd (SED 2 in Table A1 of Baron \& Netzer 2019; see also Netzer 2013), although we note that the shape of the ionizing SED has a negligible effect on our conclusions in this section. The assumed metallicity, on the other hand, has a significant effect on the derived densities, which vary by a factor of $2-3$ for a metallicity range of $0.5-2$ times solar. We present in Sec. 4.5 evidence that the metallicity is close to solar, and thus we assume solar metallicity. We considered a model grid with hydrogen densities: $\log n_{H}=2.0,2.5,3.0,3.5,4.0,4.5$, and 5.0 , and examined eight values for ionization parameter $U$ ranging from -3.8 to -2 . The separate grids in ionization parameters were created to match each of those calculated for our AGN (see Sec. 4.3 and Table 4), which were derived from the $[\mathrm{OIII}] / \mathrm{H} \beta$ and $[\mathrm{NII}] / \mathrm{H} \alpha$ ratios as described in Sec. 4.3 following Baron \& Netzer (2019). Finally, we adopted the extinction law of Cardelli (1989), taking $A_{V}=3.1 E(B-V)$ and assuming the dust is in a foreground screen. The photoionization models we consider are dusty, and thus dust is also mixed with the ionized gas (see Baron \& Netzer 2019 for additional details). However, its effect is taken into account internally within the models (we use emergent line luminosities), and thus we do not need to account for it separately. In addition, the column density of the internal dust is small compared to the column derived for the dusty screen in our sources. Two of the resulting model grids are shown in Fig. 8, for $\log U=-2.5$ and -3.5 , representing the range of $U$ we find for the individual AGN. The location of our AGN with respect to these models is shown in Fig. 8, and the implied $n_{e}$ and $A_{V}$ are given in Table 3. The median density is $n_{e}=1900 \mathrm{~cm}^{-3}$ and the $1 \sigma$ range covers 1200 $3000 \mathrm{~cm}^{-3}$, significantly higher than that derived with the doublet method.

As a consistency check of our photoionization modelling, we compare the resulting extinction to that derived from the $\mathrm{H} \alpha$ and $\mathrm{H} \beta$ lines, assuming an intrinsic ratio $\mathrm{H} \alpha / \mathrm{H} \beta=3.1$ appropriate for the narrow line region and using the Cardelli (1989) extinction curves as above (and taking into account the BLR as described in Sec. 2.5). The resulting extinction, in the range $1-3 \mathrm{mag}$, are compared in Fig. 9. Here, the dashed line indicates a 1:1 ratio, while the dotted lines are offset by $0.5 \mathrm{mag}$ each. The values from the two methods are comparable to within about $0.5 \mathrm{mag}$, providing support for our derivation of $n_{e}$ and $A_{V}$ from the [OII] and $[\mathrm{SII}]$ ratios.

\subsection{Ionization Parameter method}

The $\log U$ method was proposed by Baron \& Netzer (2019) and is based on the definition of the ionization parameter, the number of ionising photons per atom, $U=$ $Q_{L y c} / 4 \pi r^{2} c n_{H}$ where $Q_{L y c}$ is the ionising photon rate from a source, $r$ is the distance from that source, and $n_{e} \sim n_{H}$ is the local number density of atoms or equivalently electrons. The speed of light, $c$, is included to make the parameter dimensionless. These authors showed that one can 
Table 4. Measurements of the $\mathrm{H} \alpha$ and $\mathrm{H} \beta$ fluxes, the $[\mathrm{NII}] / \mathrm{H} \alpha$ and $[\mathrm{OIII}] / \mathrm{H} \beta$ line ratios, and the derived ionization parameters and densities for active galaxies

\begin{tabular}{|c|c|c|c|c|c|c|c|}
\hline Object & 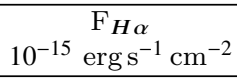 & $\begin{array}{c}\mathrm{F}_{H \beta} \\
10^{-15} \mathrm{erg} \mathrm{s}^{-1} \mathrm{~cm}^{-2} \\
\end{array}$ & $\begin{array}{c}\mathrm{A}_{V}(\mathrm{H} \alpha / \mathrm{H} \beta) \\
\operatorname{mag} \\
\end{array}$ & $\log [\mathrm{NII}] / \mathrm{H} \alpha$ & $\log [\mathrm{OIII}] / \mathrm{H} \beta$ & $\begin{array}{l}\log \mathrm{U} \\
\mathrm{cm}^{-3}\end{array}$ & $\begin{array}{l}\log n_{e} \\
\mathrm{~cm}^{-3}\end{array}$ \\
\hline ESO 137-G034 & $49.4 \pm 0.6$ & $9.25 \pm 0.13$ & 1.7 & $0.044 \pm 0.005$ & $1.091 \pm 0.006$ & $-2.64 \pm 0.01$ & $2.86 \pm 0.01$ \\
\hline MCG-05-23-016 & $6.8 \pm 0.1$ & $1.60 \pm 0.02$ & 1.0 & $-0.043 \pm 0.005$ & $1.112 \pm 0.006$ & $-2.58 \pm 0.01$ & $3.68 \pm 0.01$ \\
\hline NGC 2110 & $41.1 \pm 0.5$ & $7.85 \pm 0.10$ & 1.7 & $0.138 \pm 0.005$ & $0.678 \pm 0.005$ & $-3.31 \pm 0.01$ & $4.63 \pm 0.01$ \\
\hline NGC 2992 & $10.2 \pm 0.2$ & $1.45 \pm 0.07$ & 2.5 & $0.021 \pm 0.005$ & $1.067 \pm 0.002$ & $-2.68 \pm 0.03$ & $2.78 \pm 0.03$ \\
\hline NGC 3081 & $68.2 \pm 0.2$ & $17.8 \pm 0.4$ & 0.7 & $-0.035 \pm 0.001$ & $1.117 \pm 0.010$ & $-2.57 \pm 0.02$ & $3.49 \pm 0.02$ \\
\hline NGC 5506 & $173 \quad \pm 45$ & $26.3 \pm 0.5$ & 2.4 & $-0.052 \pm 0.118$ & $0.948 \pm 0.008$ & $-2.87 \pm 0.04$ & $4.03 \pm 0.04$ \\
\hline NGC 5728 & $41.8 \pm 1.8$ & $10.4 \pm 0.3$ & 0.8 & $0.144 \pm 0.018$ & $1.114 \pm 0.011$ & $-2.62 \pm 0.02$ & $3.48 \pm 0.02$ \\
\hline NGC 7582 & $60.9 \pm 0.3$ & $9.39 \pm 0.08$ & 2.3 & $-0.173 \pm 0.002$ & $0.362 \pm 0.003$ & $-3.54 \pm 0.01$ & $4.83 \pm 0.02$ \\
\hline ESO 021-G004 & $0.7 \pm 0.03$ & $0.09 \pm 0.01$ & 3.1 & $0.532 \pm 0.019$ & $0.886 \pm 0.046$ & $-3.02 \pm 0.07$ & $3.10 \pm 0.07$ \\
\hline NGC 1365 & $2.7 \pm 0.04$ & $0.51 \pm 0.01$ & 1.7 & $0.006 \pm 0.006$ & $0.707 \pm 0.010$ & $-3.24 \pm 0.01$ & $3.70 \pm 0.01$ \\
\hline NGC 7172 & $3.1 \pm 0.06$ & $0.36 \pm 0.02$ & 3.2 & $0.087 \pm 0.008$ & $0.799 \pm 0.019$ & $-3.14 \pm 0.03$ & $4.01 \pm 0.03$ \\
\hline
\end{tabular}

re-arrange this to give the electron density $n_{e}$ in terms of the AGN luminosity, the distance from the AGN, and the ionization parameter such that $n_{H} \propto L_{A G N} r^{-2} U^{-1}$. At the same time, they showed that $U$ can be derived rather reliably (i.e. with an uncertainty of 0.1 dex) from the strong line ratios $\mathrm{N}[\mathrm{II}] / \mathrm{H} \alpha$ and $[\mathrm{OIII}] / \mathrm{H} \beta$. That these lines are readily measurable for many AGN makes the method widely applicable.

For our sample, the absorption corrected $14-195 \mathrm{keV}$ luminosity has been calculated by Ricci et al. (2017) based on $0.3-150 \mathrm{keV}$ broadband $\mathrm{X}$-ray data. Taking those values, adjusted to our adopted distances, we have used the relations in Winter et al. (2012) to recover the AGN bolometric luminosity. Uncertainties due to both $\mathrm{X}$-ray variability and absorption correction propagate directly into the derived density; but errors in the distance to the source do not affect the derived density because the impact when converting flux to luminosity for $L_{A G N}$ is cancelled by the $1 / r^{2}$ term when converting aperture size from arcseconds to parsecs. We have already shown the line ratios in Fig. 4, and we report their values in Table 4 . These differ from those reported in Burtscher et al. (2020) by typically $<10 \%$, which is attributable to the different resolution and use of single versus multiple Gaussian profiles adopted. Also given in Table 4 is $\log U$, derived using the equations in Baron \& Netzer (2019). The final parameter is the distance $r$ from the AGN. It is clear that this method is most suited to spatially resolved data, where one can take a measurement at a known projected distance from the AGN. In our case, we have only an aperture centered on the AGN, and the luminosity distribution within this can be complex. For the purposes of this analysis, we aim to estimate a reasonable upper limit to $r$ which will therefore lead to a estimate of $n_{e}$ that is towards the lower end of the likely range. As the projected radius we therefore take the distance of $0.9^{\prime \prime}$ from the centre to the edge of the aperture, which typically corresponds to $\sim 150 \mathrm{pc}$ (this yields $r$ a factor 1.3 higher than it would be under the assumption of a uniform luminosity distribution). To account for the projection effects, we note that the AGN are Seyfert 2 (see Table 1), and hence oriented more towards edge-on than face-on. For those objects in our sample for which the orientation of the ionized outflow has been estimated, it lies in the range $10^{\circ}-49^{\circ}$ for the Sy $2 \mathrm{~s}$ from edge-on, and a slightly higher $55^{\circ}$ for the Sy 1.8 (Hjelm \& Lindblad 1996; Friedrich et al. 2010; Müller-Sánchez et al.
2011; Fischer et al. 2013; Shimizu et al. 2019). We therefore adopt an inclination of $45^{\circ}$ from edge-on (this yields $r$ a factor 1.4 higher than it would be for a fully edge-on outflow). We emphasize that in both cases, uncertainties are likely to be towards smaller values of $r$ and hence higher values for $n_{e}$ than those we derive (specifically, a reasonable range for $n_{e}$ would include values a factor 3-4 higher, but not lower). The resulting densities are reported in Table 4 . They have a median value of $4800 \mathrm{~cm}^{-3}$ and a $1 \sigma$ range of $1050-22000 \mathrm{~cm}^{-3}$. Given the large scatter in values, this is consistent with that found using the TA method, and again much higher than that derived with the doublet method. It is likely that the scatter is an observational effect related to our use of aperture measurements within which the characteristic distance from the AGN to the line emitting gas is not known. This is not a limitation of the method itself, but the impact of the way we apply it here. With aperture measurements rather than spatially resolved data, this uncertainty is particularly acute, and simply means that here we should make use of the derived densities in a statistical sense rather than focussing on individual values.

\subsection{Comparison of densities}

The distributions in Fig. 10 show that the electron densities derived for the three methods cover $1 \sigma$ ranges of: 210 $580 \mathrm{~cm}^{-3}$ for the doublet method, $1200-3000 \mathrm{~cm}^{-3}$ for the TA method, and $1050-22000 \mathrm{~cm}^{-3}$ for the $\log U$ method. The densities from both the $T A$ and $\log U$ methods are consistent, athough the scatter of the latter is wider due to observational limitations as discussed above. Both are significantly higher than the densities found with the doublet method. The reason for this difference cannot be due to saturation of the [SII] ratio at high densities, because the ratio is not close to its asymptotic limit; nor is it due to blending of the line profiles because our data are both high resolution and high signal-to-noise. Instead, it is related to the physics of the photoionization. It has been known for a long time that the hard radiation field from an AGN produces photons that can penetrate deep into clouds, leading to a partially ionized zone (Osterbrock 1989). This is much more extensive than in gas photoionized by $\mathrm{OB}$ stars, and is responsible for the excess emission from low excitation transitions seen in AGN spectra (which lead Veilleux \& Osterbrock 1987 to propose the use of species such as [SII], in terms of its flux 


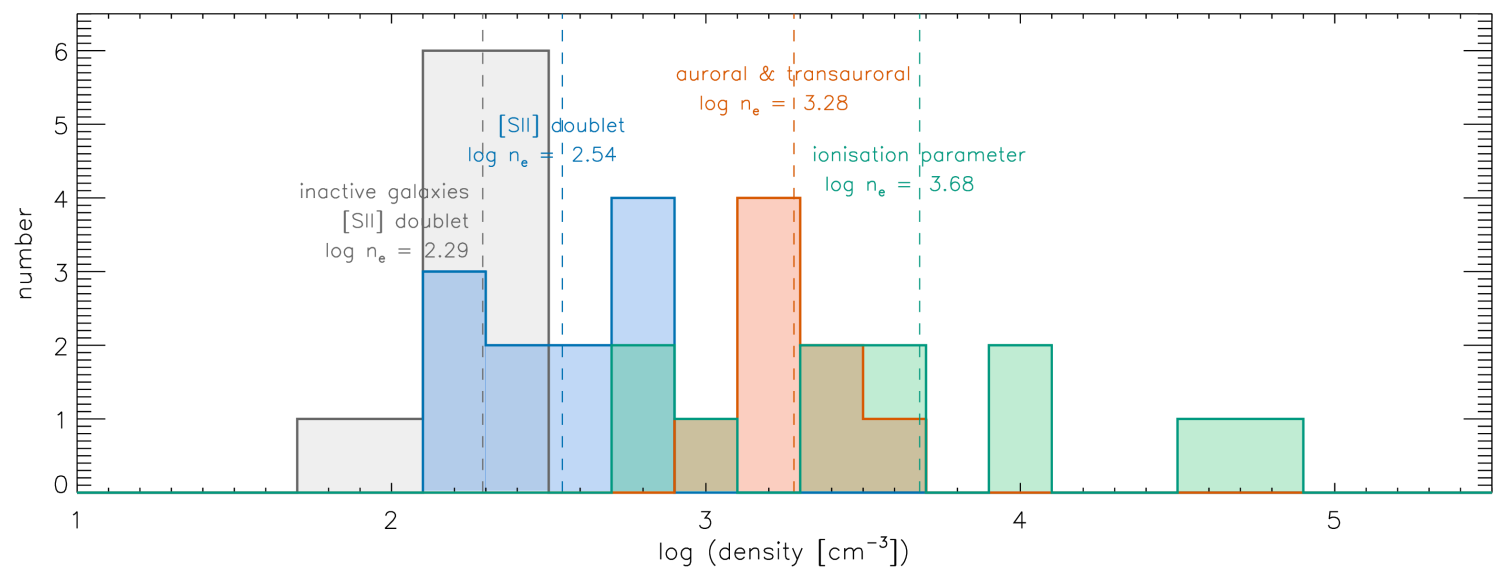

Figure 10. Comparison of the electron densities measured for the AGN using the different methods: [SII] doublet ratio (blue), using the auroral and transauroral $[\mathrm{SII}]$ and $[\mathrm{OII}]$ line ratios (red), and based on the ionization parameter (green). In addition, the inactive galaxies, for which the [SII] doublet method was used, are shown in grey.
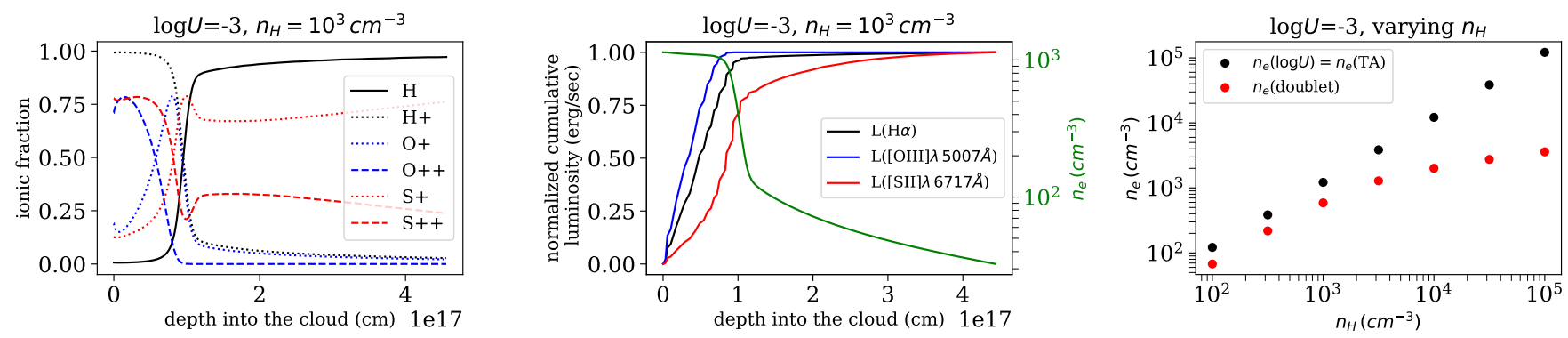

Figure 11. Photoionization model showing how different emission lines trace different properties of a constant density cloud (see Baron \& Netzer 2019 for details of the models). Left: relative population of various ions as a function of distance into the cloud. One can define the ionization front to be where most of the hydrogren is neutral. Centre: Resulting line emission, and also electron density, as a function of depth into the cloud. This shows that much of the [SII] line originates from mostly neutral gas where the electron density is lower. Right: Comparison of electron density as measured from the model using the methods described in the text, as a function of hydrogen density in the cloud. At $n_{e}>10^{3} \mathrm{~cm}^{-3}$ the discrepancy between the density derived from the [SII] doublet ratio and that found using the other methods (which trace the actual density) is significant.

ratio with respect to $\mathrm{H} \alpha$, as a diagnostic for AGN). Baron \& Netzer (2019) pointed out that because the electron density decreases further into the cloud, different excitation states of species arise from different regions within the cloud, specifically that while the $\mathrm{H} \alpha$ and [OIII] are emitted throughout most of the ionized cloud, much of the [SII] is emitted close behind the ionization front where the electron density drops dramatically. Because the ionization fraction falls rapidly below $10 \%$, most of the gas in this region is neutral.

To illustrate this point, Fig. 11 shows several results from the photoionization models. The details of these models will be given in a future publication (Baron et al. in prep.) and here we outline just the main results. In the left panel, we show the ionized fraction of hydrogen, oxygen, and sulphur, for a model with $\log U=-3$ and $\log n_{H}=3$, as a function of depth into the cloud. One can see that both $\mathrm{H}^{+}$ and $\mathrm{O}^{++}$, which are responsible for $\mathrm{H} \alpha$ and [OIII] emission respectively, peak within the ionized cloud. On the other hand, $\mathrm{S}^{+}$, which is responsible for the [SII] emission, peaks after the ionization front where more than half of the hy- drogen is already neutral. In the middle panel, we compare the cumulative line emission of $\mathrm{H} \alpha$, [OIII], and [SII], and show the electron density as a function of depth into the cloud. The $\mathrm{H} \alpha$ and [OIII] line luminosities saturate near the ionization front, and one can see that their emission traces high electron density of $n_{e} \sim 10^{3}=n_{H}$. On the other hand, the [SII] emission extends far into the neutral part of the cloud, reaching $80 \%$ of the cumulative line luminosity where the electron density has already dropped by a factor of four. Similar trends are also observed in models with different ionization parameters.

In the right panel of Fig. 11 we compare the electron densities derived using the $T A, \log U$, and doublet methods in our models with $\log U=-3$. To estimate the electron densities, we calculate the line luminosities predicted by the models and use the respective ratios discussed in Sec. 4.14.3 to estimate the electron densities, in order to match our observational approach. The electron densities derived using the $T A$ and $\log U$ methods are similar in all the models we examined, regardless of the ionization parameter. This is ex- 
Table 5. Effective Emissivities

\begin{tabular}{|c|c|c|c|}
\hline \multirow[t]{2}{*}{$\log U$} & \multicolumn{3}{|c|}{$\gamma_{e f f}{ }^{a}\left(10^{-25} \mathrm{~cm}^{3} \mathrm{erg} \mathrm{s}^{-1}\right)$} \\
\hline & $\mathrm{H} \alpha$ & [SII] $6716 \AA^{b}$ & [OIII] $5007 \AA$ \\
\hline-2.00 & 1.75 & 0.46 & 7.03 \\
\hline-2.26 & 2.02 & 0.58 & 7.41 \\
\hline-2.51 & 2.25 & 0.79 & 7.19 \\
\hline-2.77 & 2.45 & 1.13 & 6.35 \\
\hline-3.03 & 2.57 & 1.60 & 4.81 \\
\hline-3.29 & 2.53 & 2.12 & 2.87 \\
\hline-3.54 & 2.55 & 2.86 & 1.33 \\
\hline-3.80 & 2.26 & 3.17 & 0.41 \\
\hline
\end{tabular}

a See Sec. 4.5 for an explanation of how these were calculated.

$b$ The values for [SII] emissivity are only valid for $n_{e}<10^{3} \mathrm{~cm}^{-3}$.

pected since both methods trace the hydrogen density in the cloud, which is assumed to be constant in our models. We find a significant difference between the electron densities derived using either of those methods, and those derived using the doublet method. This difference increases as the hydrogen density in the cloud increases, and becomes significant even below the critical density $n_{H} \sim 10^{4} \mathrm{~cm}^{-3}$ of the [SII] lines. This is because, independent of the hydrogen density, the [SII] lines trace low electron density regions within the ionized cloud. We therefore suggest that the doublet-based electron densities provide a biased view of the ionized cloud, and that they should not be used above $n_{e} \sim 10^{3} \mathrm{~cm}^{-3}$, since then the hydrogen density (and thus the electron density in the ionized part of the cloud) can be a factor of 3-100 larger. This invalidates key assumptions in the use of the [SII] doublet to estimate the density of the ionized cloud, and thus its mass.

\subsection{Ionized gas masses}

As noted in Sec. 1, given a particular transition with volume emissivity $\gamma_{\text {line }}$ and emission line luminosity $L_{\text {line }}$, the ionized gas mass can be estimated using $M_{i o n}=$ $\mu m_{H} L_{\text {line }} /\left(\gamma_{\text {line }} n_{e}\right)$, where $n_{e}$ is the electron density in the line-emitting region. The volume emissivity, $\gamma_{\text {line }}$, depends on known atomic physics and on the physical properties of the cloud (for additional details see Baron \& Netzer 2019). We have demonstrated in Sec. 4.4 that different emission lines are emitted in different parts of the ionized cloud, with those regions showing significantly different electron densities and temperatures. Therefore, the common practice of estimating the ionized gas mass using $L_{H \alpha}$ or $L_{[O I I I]}$ and an electron density from the [SII] doublet is invalid and will result in an over-estimate of the ionized gas mass. As first argued by Baron \& Netzer (2019), it is necessary to use density tracers that match the emission line in question in order to obtain an unbiased estimate of the gas mass in the cloud. For example, when using $L_{H \alpha}$ or $L_{[O I I I]}$, it is necessary to use $T A$ or $\log U$ based electron densities; when estimating the ionized gas mass using the doublet based density, it is necessary to use the [SII] luminosity (although with an additional caveat described below about the valid density range). Mixing these up would result in an incorrect estimate of the ionized gas mass.

Baron \& Netzer (2019) calculated the effective line emissivities for the $\mathrm{H} \alpha$ and [OIII] emission lines, by taking the mean emissivity in the cloud weighted by the electron density. Since, in those models, the $\mathrm{H} \alpha$ and [OIII] lines are emit- ted in similar regions within the ionized cloud, the effective emissivities are expected to result in similar masses when using either the $\mathrm{H} \alpha$ or $[\mathrm{OIII}]$ emission lines. However, if two transitions are emitted in different regions within the cloud, we no longer expect their masses to be equal to each other, even when using the appropriate effective emissivities and electron densities. This is because the mass of the gas that emits the different transitions may be different. For the example in Fig. 11, the mass of the [SII]-emitting gas is roughly a factor two larger than the mass in the $\mathrm{H} \alpha$-emitting region. More generally we find that, for $n_{e} \lesssim 10^{3} \mathrm{~cm}^{-3}$, the gas mass ratio of the [SII]-emitting and $\mathrm{H} \alpha$-emitting regions is $1-3$, depending on the ionization parameter.

In this work, our goal is to calculate effective line emissivities for the $\mathrm{H} \alpha$, [OIII], and [SII] emission lines, that will result in similar ionized gas masses. To achieve this, it is necessary to scale each of the emissivities so that the mass traced by the different transitions is similar. We begin by defining an effective electron density $\left\langle n_{e}\right\rangle_{\text {line }}$ as the luminosity weighted mean electron density in the cloud. Its value will depend on the emission line used, and is a reasonable approximation to the values that would be derived observationally using the methods discussed in Sec. 4.1-4.3. We define the ionization front, separating the fully ionized and mostly neutral regions of the cloud, to be where $80 \%$ of the hydrogen is neutral ${ }^{1}$. Defining the ionized gas mass $M_{i o n}$ to be the mass of the gas before the ionization front, we calculate the effective product $\left\langle\gamma_{\text {line }} n_{e}\right\rangle=\mu m_{H} L_{\text {line }} / M_{\text {ion }}$ using the integrated line luminosity $L_{\text {line }}$ for each of the three emission lines $\mathrm{H} \alpha$, [OIII], and [SII]. We can then define the new effective emissivities as $\left\langle\gamma_{\text {line }}\right\rangle=\left\langle\gamma_{\text {line }} n_{e}\right\rangle /\left\langle n_{e}\right\rangle_{\text {line }}$. Values for $\left\langle\gamma_{\text {line }}\right\rangle$ for $\mathrm{H} \alpha$, [OIII], and [SII] are given in Table 5 as a function of $\log U$. It is notable in this Table that even $\gamma_{H \alpha}$ is rather different from its canonical value of $3.6 \times 10^{-25} \mathrm{~cm}^{3} \mathrm{erg} \mathrm{s}^{-1}$ (Osterbrock 1989). By construction, $\left\langle\gamma_{\text {line }}\right\rangle$ will yield a similar mass for the ionized gas independent of which tracer is used, as long as one uses the matching estimate of electron density. Our constant density models verify the efficacy of this approach to $10-20 \%$.

Fig. 12 compares the ionized masses derived using this technique for our AGN sample. The far left panel shows we find a negligible $(<0.1$ dex) difference in mass when using $\mathrm{L}_{H \alpha}$ or $\mathrm{L}_{[O I I I]}$. Since these were both calculated using the same density tracer, it is a verification of our measurements and our approach to calculating $\gamma_{\mathrm{eff}}$. It also acts as a posteriori support for adopting solar metallicity. The centre left panel compares the $T A$ and $\log U$ methods for the same line. Since our models indicate that these should yield the same density, the modest difference is likely due to a systematic effect in AGN luminosity or adopted distance from the AGN to the line emission, or due to complexities that our constant density models do not address. The centre right panel shows a factor three difference between using the [OIII] line with the TA method, and the [SII] line with the doublet method. The former is more robust, and as indicated in the right panel of Fig. 11, the difference between this and the [SII] derived mass is a further indication that $n_{e}>10^{3} \mathrm{~cm}^{-3}$

1 The exact definition of the ionization front (i.e., where the $50 \%$, $80 \%$, or $90 \%$ of the hydrogen is neutral) has a negligible effect on the resulting ionized gas mass 

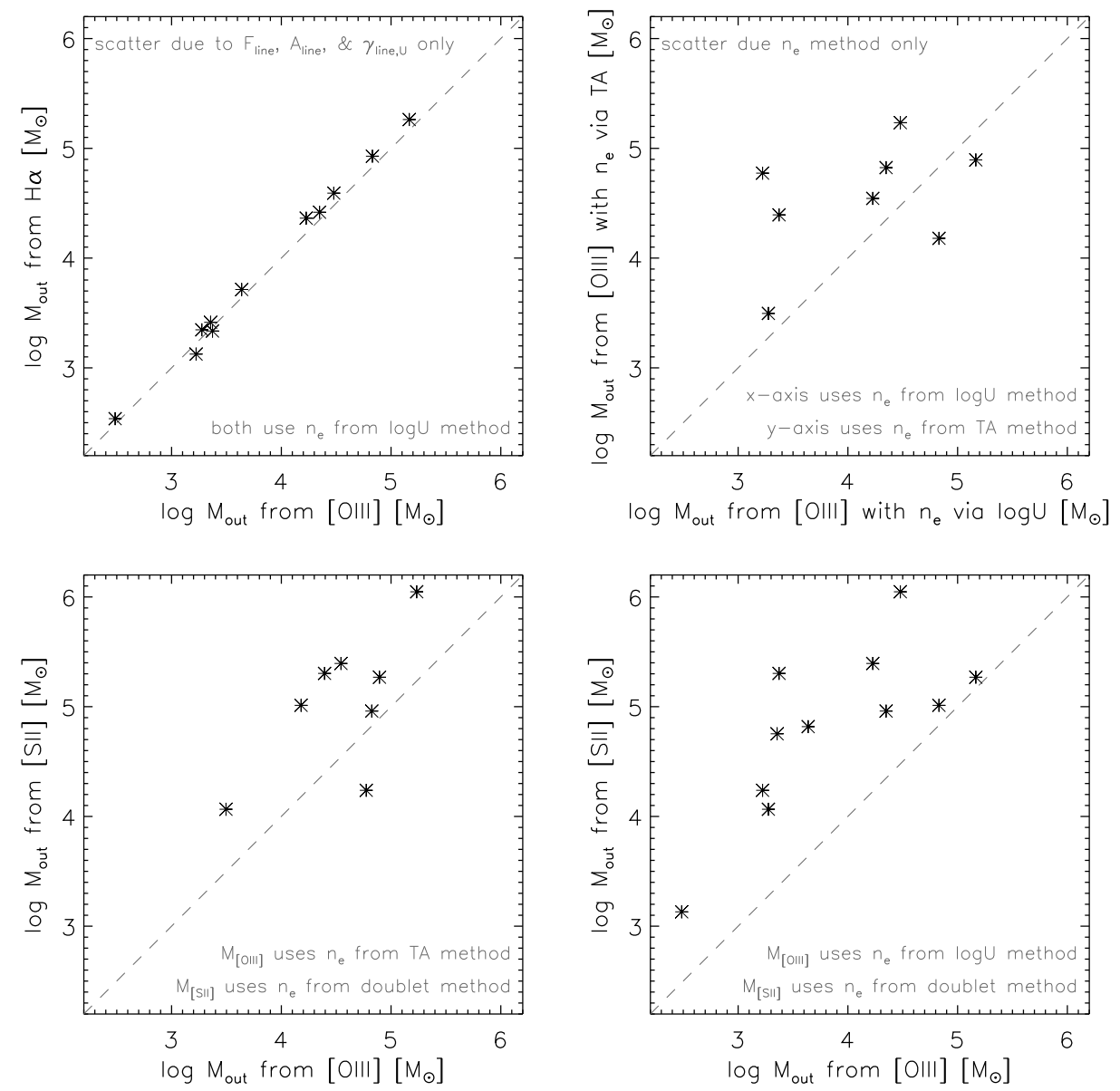

Figure 12. Comparison of the derived masses using different tracers and density estimates. Top left: when using the same $\mathrm{n}_{e}$ method, the masses from the $\mathrm{H} \alpha$ line and [OIII] line are very similar. Top right: when using the same line, the masses using $\mathrm{n}_{\boldsymbol{e}}$ from the $\log U$ and $T A$ methods scatter around a 1:1 line. Bottom: an offset is found for the masses derived from the [SII] line with $\mathrm{n}_{e}$ from the doublet method, and the [OIII] line with $\mathrm{n}_{e}$ from the TA (left) or $\log U$ (right) method. This offset is consistent with the rapid increase in discrepancy for the doublet method at $n_{e}>10^{3} \mathrm{~cm}^{-3}$, where our calculation of $\gamma_{[S I I]}$ is no longer valid. See the text for a discussion of these panels.

where our calculated $\gamma_{[S I I]}$ can no longer be properly applied. Similarly, the far right panel compares the [OIII] line and $\log U$ method with the [SII] line and doublet method.

Our summary for these three density estimators is then: The doublet method is severely biased. It will yield incorrect masses unless used with the [SII] line luminosity and $\gamma_{[S I I]}$ given in Table 5; and even then it can only be used when $n_{e}<10^{3} \mathrm{~cm}^{-3}$. The TA method is robust, but unfortunately in many cases impractical because it relies on measurements of very weak lines. Even in our sample of local luminous AGN we were unable to use it in about $1 / 3$ of the cases. The $\log U$ method is both robust and straightforward to apply. It has the additional advantage of emphasizing, by its definition, that the density is expected to decrease at increasing distance from the AGN. It is, however, more suited to spatially resolved data and one needs to be careful when estimating the characteristic distance from the AGN to the outflow in aperture measurements. It is this method, together with the [OIII] line luminosity and respective emissivity that we use in Sec. 5 when estimating the outflow rates for the AGN.

\section{OUTFLOW MASS AND RATE}

To estimate the outflow rate we have adopted the standard relation $\dot{M}_{\text {out }}=M_{\text {out }} v_{\text {out }} / r_{\text {out }}$ which is valid for an outflow rate that is constant over time, a reasonable assumption to make since the flow timescales in question are $<0.5 \mathrm{Myr}$. The same expression is also valid for the time averaged thin shell approach (Lutz et al. 2020). Similarly the kinetic power of the outflow is calculated as: $\dot{E}_{\text {kin }}=1 / 2 \dot{M}_{\text {out }} v_{\text {out }}^{2}$.

We have used the [OIII] line luminosity when calculating the outflowing mass, and adopted the volume emissivity $\gamma_{[O I I I]}$ given in Table 5. To be consistent, we have also used an outflow velocity $v_{98,[O I I I]}$. In terms of $n_{e}$, we have taken that derived using the $\log U$ method. The resulting outflowing masses and rates are given in Table 6 . We note that if we were to use the $\mathrm{H} \alpha$ line luminosity to estimate mass, with $\gamma_{H \alpha}$ as given in Table 5, we would get very similar outflowing masses as is shown in the left panel of Fig. 12; and if we were to use $v_{98,[S I I]}$ as the outflow velocity, the left panel of Fig. 3 shows that the the outflow rates would be about $25 \%$ lower. As a cross-check, we compare the outflow rates for 
Table 6. Measured and derived properties related to outflows in the active galaxies.

\begin{tabular}{|c|c|c|c|c|c|c|c|c|}
\hline Object & $\begin{array}{c}\log \mathrm{L}_{A G N} N \\
\operatorname{erg~s}^{-1}\end{array}$ & $\begin{array}{c}\mathrm{R}_{a p} \\
\mathrm{pc}\end{array}$ & $\begin{array}{l}\log \mathrm{L}_{[\text {OIIII }]} \\
\quad \operatorname{erg~s}^{-1}\end{array}$ & 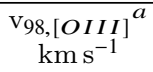 & $\begin{array}{c}\log M_{\text {out }} b \\
\mathrm{M}_{\odot}\end{array}$ & $\begin{array}{c}\log \dot{M} \\
\mathrm{M}_{\odot} \mathrm{yr}^{-1}\end{array}$ & $\begin{array}{c}\dot{E}_{k i n} \\
\operatorname{erg~s}^{-1}\end{array}$ & $\log \dot{E}_{k i n} / L_{A G N}$ \\
\hline ESO 137-G034 & 43.4 & 152 & 40.8 & 517 & 5.2 & 0.49 & 40.6 & -2.8 \\
\hline MCG-05-23-016 & 44.3 & 152 & 39.7 & 252 & 3.3 & 0.003 & 37.8 & -6.5 \\
\hline NGC 2110 & 44.5 & 148 & 40.4 & 1665 & 3.4 & 0.03 & 40.4 & -4.1 \\
\hline NGC 2992 & 43.3 & 157 & 40.4 & 587 & 4.8 & 0.27 & 40.5 & -2.8 \\
\hline NGC 3081 & 44.1 & 148 & 40.6 & 279 & 4.3 & 0.04 & 39.6 & -5.0 \\
\hline NGC 5506 & 44.1 & 117 & 41.2 & 792 & 4.5 & 0.21 & 40.6 & -3.5 \\
\hline NGC 5728 & 44.1 & 170 & 40.5 & 875 & 4.2 & 0.09 & 40.3 & -3.8 \\
\hline NGC 7582 & 44.0 & 95 & 40.1 & 364 & 3.2 & 0.007 & 38.5 & -5.6 \\
\hline ESO 021-G004 & 43.3 & 170 & 39.4 & 432 & 3.6 & 0.01 & 38.8 & -4.5 \\
\hline NGC 1365 & 43.0 & 78 & 38.6 & 338 & 2.5 & 0.001 & 37.7 & -5.4 \\
\hline NGC 7172 & 44.1 & 161 & 39.9 & 397 & 3.3 & 0.006 & 38.4 & -5.6 \\
\hline
\end{tabular}

$a$ For consistency with our use of $\mathrm{L}_{[\boldsymbol{O I I I}]}$, we have used $v_{98,[\boldsymbol{O I I I}]}$ rather than $v_{98,[S I I]}$ although typically they differ by only $25 \%$.

$b$ As discussed in Sec. 4.4 we have adopted $n_{e}$ derived using the $\log U$ method.

two objects to recently published values based on spatially resolved data. Despite the different methods used, our outflow rate for NGC 5728 compares well to that of $0.08 M_{\odot} \mathrm{yr}^{-1}$ for both sides of the outflow within $250 \mathrm{pc}$ found by Shimizu et al. (2019). For NGC 1365, the outflow rate on the southeast side within $380 \mathrm{pc}$ found by Venturi et al. (2018) was $0.01 M_{\odot} \mathrm{yr}^{-1}$. This is about a factor 10 higher than ours because, as discussed above, the density from the [SII] doublet used by those authors, leads to an overestimate of the outflowing mass by that amount.

\subsection{Outflow rate relation at low luminosity}

Although the AGN in our sample are considered luminous with respect to local AGN, they are of only moderate luminosity when compared to samples taken from larger distances that include luminous quasars. As such, they provide an opportunity to extend to lower luminosity the relation between AGN luminosity and outflow rate in the literature. One of the most well known is that of Fiore et al. (2017), which is shown in the left panel of Fig. 13. However, there are two important adjustments to our data required in order to effect such a comparison. The first is that Fiore et al. (2017) adopted a definition of outflow rate that is a factor three higher than the one we use above (which is appropriate for an outflow which fills the volume with a constant average density). The second is that to deal with the multitude of values for $n_{e}$ adopted in the literature by different authors in the data they compiled, Fiore et al. (2017) re-scaled the mass of outflowing gas using a single electron density of $n_{e}=200 \mathrm{~cm}^{-3}$. This is a rather problematic issue, because that density is less than any of those we have measured in our AGN sample. To indicate the major impact this has, we have plotted our derived outflow rates from Table 6 as filled circles, and values adjusted to $n_{e}=200 \mathrm{~cm}^{-3}$ as open circles. It is immediately clear that, the relatively low scatter in the relation over 5 orders of magnitude is at least partly due to the adoption of a single value for the density of all the outflows. Using the densities we have derived, the low luminosity AGN are highly scattered and lie below where one would expect from the correlation.

Addressing this issue has prompted us to look also at the parameters $L_{\text {line }}$ and $v_{\text {out }}$ that contribute to the relation given that, for constant $n_{e}, \dot{M}_{\text {out }} \propto L_{\text {line }} v_{\text {out }} / r_{\text {out }}$ as shown in Sec. 1 (and noting that there is no individual correla- tion between $L_{A G N}$ and $r_{\text {out }}$ ). Using the data in Fiore et al. (2017), we have calculated (by 'reverse engineering') the effective [OIII] luminosity $L_{[O I I I], \text { eff }}$ that would be required to produce the given outflow rate, and have verified this approach using the [OIII] luminosities available (F. Fiore priv. comm.). The individual correlations for $L_{[O I I I], \text { ef } f}$ and $v_{\text {out }}$ are shown in the centre and right panels of Fig. 13. They show that it is the line luminosity $L_{[O I I I]}$ rather than the outflow speed $v_{\text {out }}$ that must be the primary driver of the correlation over so many orders of magnitude. It is a long established relation for X-ray selected AGN (Mulchaey et al. 1994; Alonso-Herrero et al. 1997; Heckman \& Best 2014), which has a similar large scatter. Using a sample selected on the basis of broad $\mathrm{H} \alpha$, Stern \& Laor (2012) find a good (approximately linear) correlation with a scatter of 0.44 dex; and based on compiled data for a very hard X-ray selected sample, Berney et al. (2015) suggest that the scatter is 0.62 dex. The scatter for the Fiore et al. (2017) data shown here is 0.67 dex. Although there will be additional uncertainty in our 'reverse engineered' estimate of $\mathrm{L}_{[O I I I]}$, the correlation covers the range required, and naturally accounts for the approximate proportionality between $\dot{M}_{\text {out }}$ and $\mathrm{L}_{A G N}$ - although some authors have noted a non-linear relation between narrow line and AGN luminosity (Netzer et al. 2006; Stern \& Laor 2012).

Given the issues above, we explore an alternative representation of the Fiore et al. (2017) relation: instead of fixing a constant density for all objects, we adopt an estimate of the density based on the ionization parameter method. We use the given AGN luminosites $L_{A G N}$ and outflow sizes $r_{\text {out }}$, making the same assumptions as in Sec. 4.3 about distribution of line luminosity within an aperture and projection. We adopt a value of $\log U=-2.7$, calculated to be the median for the outflows in Fiore et al. (2017) for which both [OIII] and $\mathrm{H} \beta$ luminosities are available (F. Fiore priv. comm.), and which should therefore be characteristic for AGN photoionized outflowing gas. We can then make an order-ofmagnitude estimate of $n_{e}$ as illustrated in Fig. 14. The key point of this figure is that it emphasizes the enormous variation that can be expected for $n_{e}$ in different outflows. It highlights how, in the central few hundred parsecs one can find densities in the range $10^{3}-10^{4} \mathrm{~cm}^{-3}$; and that while in some AGN this may fall to $\sim 100 \mathrm{~cm}^{-3}$ at kiloparsec scales, for luminous quasars it may still be in the $\sim 1000 \mathrm{~cm}^{-3}$ range.

Bearing in mind the expected variation in outflow den- 

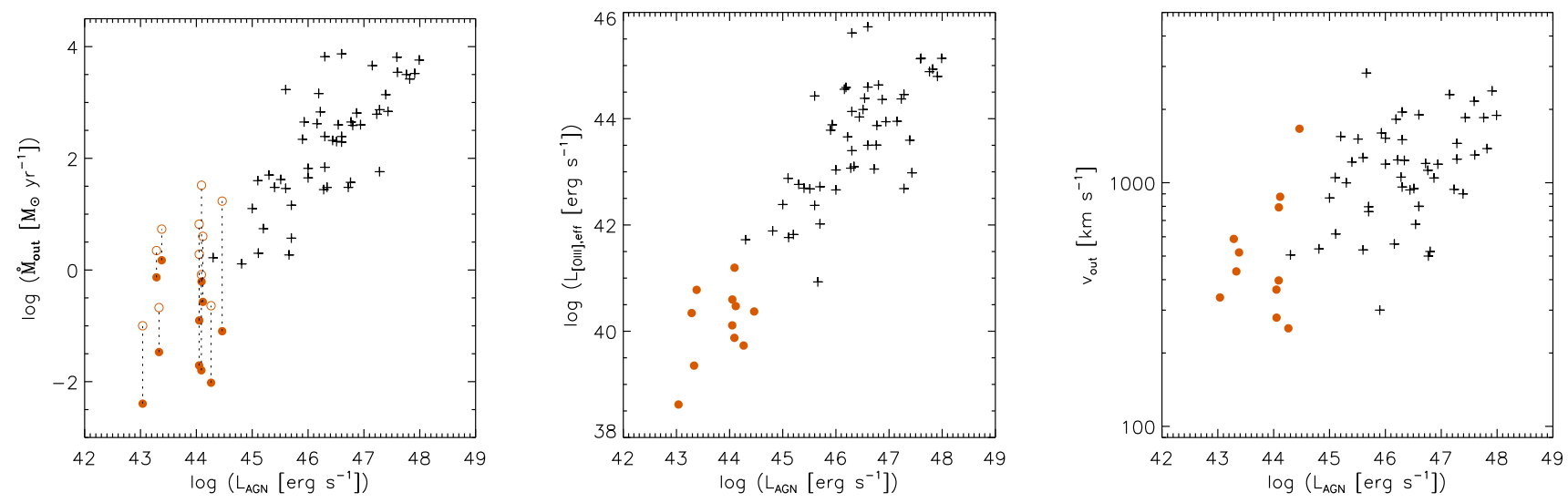

Figure 13. Relations with $L_{A G N}$ using data from Fiore et al. (2017) (black plus points) and from Table 6 for our AGN sample (red filled circles). Left: relation with $\dot{M}_{\text {out }}$. In this panel, the outflow rates for our AGN have been increased by a factor three to match the equation used by Fiore et al. (2017). The open circles indicate how those points would shift if in addition we used $n_{e}=200 \mathrm{~cm}^{-3}$ as adopted by those authors for all their objects. One needs to be cautious with this relation. Centre: relation with $L_{[O I I I], e f f}$, the luminosity that would be required to produce the given outflow rate. This covers 5 orders of magnitude, and is the primary driver of the outflow rate relation. Right: relation with $v_{\text {out }}$ (as given by Fiore et al. 2017, and $v_{98,[O I I I]}$ for our AGN). This covers barely a single order of magnitude and so does not contribute much to the outflow rate relation.

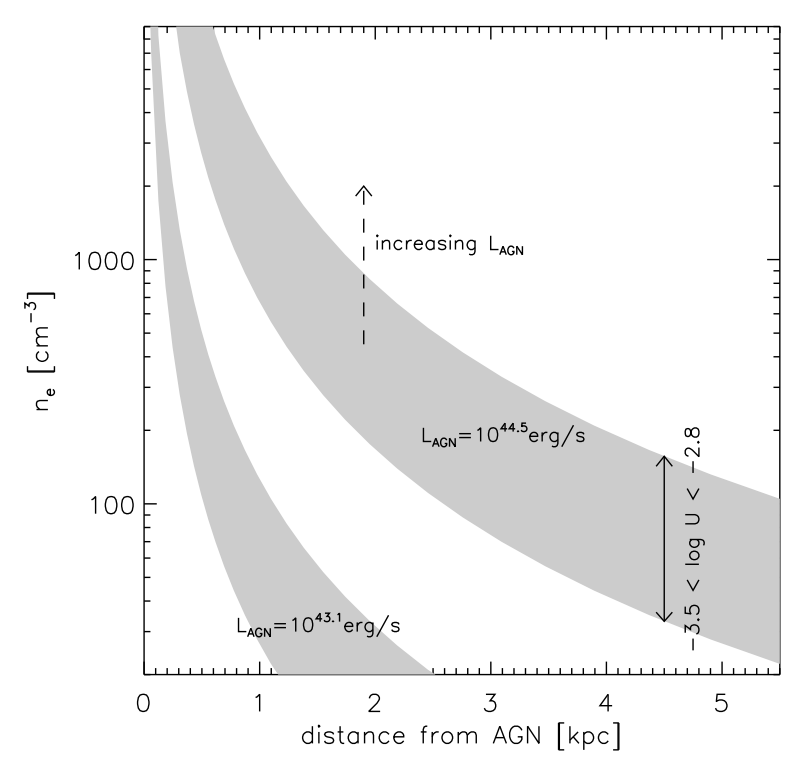

Figure 14. Illustration of how one might estimate a reasonable range for the electron density in an outflow at a given distance from an AGN of known luminosity. This figure shows two example ranges for $L_{A G N}$ corresponding to the minimum and maximum in our sample. The grey regions indicate the uncertainty due to an unknown ionization parameter. This figure should be used as a guide only, and emphasizes how much variation can be expected in outflow densities between objects and at different radii.

sity, in Fig. 15 we have modified the relation of Fiore et al. (2017) to take into account our best estimate of $n_{e}$ for the individual objects, and overplotted both our data as well as that from Baron \& Netzer (2019). The relation between AGN luminosity and outflow rate remains, but with two im-

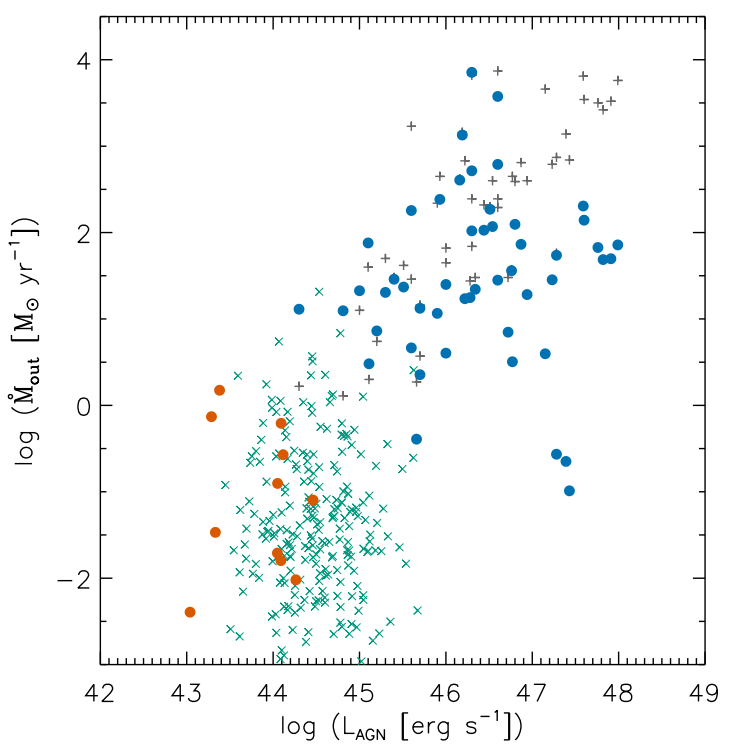

Figure 15. A modification to the well known relation from Fiore et al. (2017) in which we have adjusted the outflow rates according to our best estimate of the density. The original data are shown as grey plus points, while the adjusted points are drawn as filled blue circles. We have included our own points as filled red circles (again a factor three more than in Table 6, as described in Sec. 5.1), as well as the data from Baron \& Netzer (2019) as green crosses. A correlation remains but there is more scatter than before, and with the original data tracing its upper limit. The three outliers at high $L_{A G N}$ and low $\dot{M}$ are at $z \sim 2.4$. One was already a low point on the original relation; the other two have very high estimated densities because $r_{\text {out }}$ is small. 
portant differences: (i) the scatter is much larger than before, and (ii) the outflow rates are typically lower by about a factor three since the densities are generally that much higher. The original relation appears to be more of an upper limit on the outflow rate, but not all AGN driven outflows reach that limit. With the data available, the values we have estimated for $n_{e}$ are still uncertain and so we would only claim that this modified relation should be considered as equally valid as the original relation of Fiore et al. (2017). It does, however, underline that there remain unanswered questions about how the ionized gas outflow rate is related to AGN luminosity.

\section{CONCLUSION}

We present an analysis of the kinematics and electron density derived from emission lines integrated over the central $1.8^{\prime \prime}(\sim 300 \mathrm{pc})$ of local luminous active galaxies and matched inactive galaxies. The data are from Xshooter observations, taken as part of the LLAMA survey which comprises a complete volume limited sample of $14-195 \mathrm{keV}$ selected AGN together with inactive galaxies matched by their global properties. We find:

- The peak of the emission lines in the AGN are offset from systemic (traced by stellar absorption features), indicating that the line profiles of the AGN are dominated by outflow. Any systemic component is highly sub-dominant. In contrast, the line profiles of the inactive galaxies are characterised by a core close to the systemic velocity with a wing tracing outflow.

- For the galaxies with weak emission lines (equivalent width of $[\mathrm{SII}] 6716 \AA \stackrel{\sim}{<} 10 \AA$ ), the $[\mathrm{SII}]$ doublet ratio method overestimates the electron density unless a correction is made for stellar absorption features. This is particularly important for the inactive galaxies where the impact on $n_{e}$ is a factor two. After correcting for the stellar continuum, their median density is found to be $n_{e}=200 \mathrm{~cm}^{-3}$.

- For the active galaxies, the densities found using three independent methods (the [SII] doublet ratio, a method using auroral and transauroral line ratios, and a method based on the ionization parameter) differ. The median densities found with these methods are $460 \mathrm{~cm}^{-3}, 1900 \mathrm{~cm}^{-3}$, and $4800 \mathrm{~cm}^{-3}$ respectively. The latter two have comparable ranges and are both significantly larger than the density found with the [SII] doublet ratio. An explanation for this difference lies in the ionization structure of the clouds. Specifically, in the conditions considered here, most of the [SII] line emission originates in a partially ionized zone where $n_{e}$ drops rapidly to below $10 \%$ of $n_{H}$ and most of the gas is neutral. This invalidates the assumptions under which the [SII] doublet is used to estimate density.

- The definition of 'ionized mass' ought to be treated carefully. Specifically, the extensive region in which most of the gas is neutral increases the total mass of the cloud by a factor $2-4$ with respect to the fully ionized gas.

- The implied outflow rates are $0.001-0.5 \mathrm{M}_{\odot} \mathrm{yr}^{-1}$, and span an order of magnitude more range than the AGN luminosities of $(1-30) \times 10^{43} \mathrm{erg} \mathrm{s}^{-1}$. For these moderate luminosity AGN, there is no apparent relation between the two quantities.
- The AGN in this sample extend the lower luminosity end of the relation between outflow rate and AGN luminosity in the literature. However, caution needs to be applied when making such comparisons because of uncertainties in the outflow density. Adjusting the data points in the relation using our best estimate of $n_{e}$ shows that there may be more scatter than previously thought, and that the outflow rates may be at least a factor of a few lower than previously estimated.

\section{ACKNOWLEDGEMENTS}

We thank all the staff of the Paranal Observatory who were involved in carrying out the service mode observations used in this paper. And we extend particular thanks to Fabrizio Fiore for providing additional data for the ionised outflows. We also thank Marina Trevisan for a discussion about features in the stellar continuum. R.R. thanks CNPq, CAPES, and FAPERGS. This research has made use of the NASA/IPAC Extragalactic Database (NED), which is funded by the National Aeronautics and Space Administration and operated by the California Institute of Technology.

\section{REFERENCES}

Alonso-Herrero A., Ward M., Kotilainen J., 1997, MNRAS, 288, 977

Bae H.-J., Woo J.-H., 2016, ApJ, 828, 97

Baron D., Netzer H., Prochaska J. X., Cai Z., Cantalupo S., et al., 2018, MNRAS, 480, 3933

Baron D., Netzer H., 2019, MNRAS, 486, 4290

Baron D., Netzer H., Davies, R., Prochaska J.X., 2020, MNRAS, submitted

Baumgartner W., Tueller J., Markwardt C., Skinner G., Barthelmy S., et al., 2013, ApJS, 207, 19

Berney S., Koss M., Trakhten B., Ricci C., Lamperti I., et al., 2015, MNRAS, 454, 3622

Binggeli B., Sandage A., Tammann G., 1985, AJ, 90, 1681

Brinchmann J., Charlot S., Whilte S., Tremonit C., Kauffmann G., Heckman T, Brinkmann J., 2004, MNRAS, 351, 1151

Burtscher L., et al., 2020, in prep.

Buta R., Sheth K., Athanassoula E., Bosma A., Knapen J., et al., 2015, ApJS, 217, 328

Caglar T., Burtscher L., Brandl B., Brinchmann J., Davies R., et al., 2020, A\&A, accepted

Cappellari M., Emsellem E., 2004, PASP, 116, 138

Cappellari M., 2017 MNRAS, 466, 798

Cardelli J., Clayton C., Mathis J., 1989, ApJ, 345, 245

Carniani S., Marconi A., Maiolino R., Balmaverde B., Brusa M., et al., 2015, A\&A, 580, A102

Cid Fernandes R., Stasińska G., Schlickmann M., Mateus A., Vale Asari N., Schoell W., Sodré Jr L., 2010, MNRAS, 403, 1036

Cid Fernandes R., Stasińska G., Mateus A., Vale Asari N., 2011, MNRAS, 413, 1687

Coelho, P., 2014, MNRAS, 440, 1027

Davies R., Burtscher L., Rosario D., Storchi-Bergmann T., Contursi A., et al., 2015, ApJ, 806, 127

Davies R., Hicks E., Erwin P., Burtscher L., Contursi A., et al., 2017, MNRAS, 466, 4917

De Vaucouleurs G., de Vaucouleurs A., Corwin Jr, H., Buta R., Paturel G., Fouque P., 1991, in Third Reference Catalogue of Bright Galaxies

Doyle M., Drinkwater M., Rohde D., Pimbblet K., Read M., et al., 2005, MNRAS, 361, 34 
Do Nascimento J., Storchi-Bergmann T., Mallmann N., Riffel R., Ilha G., et al., 2019, MNRAS, 486, 5075

Fabian A., 2012 ARA\&A, 50, 455

Ferland G., Chatzikos M., Guzmán F., Lykins M., van Hoof P., et al., 2017, RMxAA, 53, 385

Fiore F., Feruglio C., Shankar F., Bischetti M., Bongiorno A., et al., 2017, A\&A 601, A143

Fischer T., Crenshaw D.M., Kraemer S., Schmitt H., 2013, ApJSS, 209, 1

Fischer T., Machuca C., Diniz M., Crenshaw D.M., Kraemer S., et al., 2017, ApJ, 834, 30

Fischer T., Kraemer S., Schmitt H., Longo Micchi L., Crenshaw D.M., et al., 2018, ApJ, 856, 102

Förster Schreiber N.M., Übler H., Davies R.L., Genzel R., Wisnioski E., et al., 2019, ApJ, 875, 21

Freitas I., Riffel R.A., Storchi-Bergamnn T., Elvis M., Robinson A., et al., 2018, MNRAS, 476, 2760

Friedrich S., Davies R., Hicks E., Engel H., Müller-Sánchez F., Genzel R., Tacconi L., 2010, A\&A, 519, A79

Harrison C., Alexander D., Mullaney J., Swinbank A.M., 2014 MNRAS, 441, 3306

Harrison C., Costa T., Tadhunter C., Flütsch A., Kakkad D., et al., 2018, NatAs, 2, 198

Heckman T., Best P., 2014, ARA\&A, 52, 589

Hjelm M., Lindblad P., 1996, A\&A, 305, 727

Holt J., Tadhunter C., Morganti R., Emonts B., 2011, MNRAS, 410, 1527

Kakkad D., Mainieri V., Padovani P., Cresci G., Husemann B, et al., 2016, A\&A, 592, A148

Kakkad D., Groves B., Dopita M., Thomas A., Davies R., et al., 2018, A\&A, 618, A6

Kauffmann G., Heckman T., Tremonti C., Brinchmann J., Charlot S., et al., 2003, MNRAS, 346, 1055

Kewley L., Dopita M., Sutherland R., Heisler C., Trevena J., 2001, ApJ, 556, 121

Kewley L., Groves B., Kauffmann G., Heckman T., 2006, MNRAS, 372, 961

King A., Pounds K., 2015, ARA\&A, 53, 115

Lin M.-Y., Davies R., Hicks E., Burtscher L., Contursi A., et al., 2018, MNRAS, 473, 4582

Liu G., Zakamska N., Greene J., Nesvadba N., Liu X., 2013 MNRAS, 436, 2576

Lutz D., Sturm E., Janssen A., Veilleux S., Aalto S., et al., 2020, A\&A in press

Markwardt C., 2009, in Non-Linear Least Squares Fitting in IDL with MPFIT, Proc. Astronomical Data Analysis Software and Systems XVIII, Quebec, Canada, ASP Conference Series, Vol. 411, eds. D. Bohlender, P. Dowler, D. Durand (Astronomical Society of the Pacific: San Francisco), p. 251-254

Mendel T., Proctor R., Forbes D., Brough S., 2008, MNRAS, 389, 749

Mulchaey J., Koratkar A., Ward M., 2994, ApJ, 436, 586

Müller-Sánchez F., Prieto M.A., Hicks E., Vives-Arias H., Davies R., Malkan M., Tacconi L., Genzel R., 2011, ApJ, 739, 69

Netzer H., Mainieri V., Rosati P., Trakhtenbrot B., 2006, A\&A, 453,525

Netzer H., 2009, MNRAS, 399, 1907

Netzer H., 2013, The Physics and Evolution of Active Galactic Nuclei

Osterbrock D., 1989, Astrophysics of Gaseous Nebulae and Active Galactic Nuclei (Sausalito, CA: Univ. Science Books)

Perna M., Lanzuisi G., Brusa M., Cresci G., Mignoli M., 2017, A\&A, 606, A96

Pisano D., Barnes D., Staveley-Smith L., Gibson B., Kilborn V., Freeman K., 2011, ApJS, 197, 28

Ricci C., Trakhtenbrot B., Koss M., Ueda Y., Del Vecchio I., et al., 2017, ApJSS, 233, 17

Riffel R.A., Storchi-Bergmann T., Winge C., 2013 MNRAS, 430,
2249

Riffel R.A., Storchi-Bergmann T., Riffel R., 2015, MNRAS, 451, 3587

Rose M., Tadhunter C., Ramos Almeida C., Rodríguez Zaurín J., Santoro F., Spence R., 2018 MNRAS, 474, 128

Rosario D., Burtscher L., Davies R., Koss M., Ricci C., et al., 2018, MNRAS, 473, 5658

Rupke D., Veilleux S., 2011, ApJL, 729, L27

Rupke D., Veilleux S., Sanders D., 2005, ApJS, 160, 87

Rupke D., Veilleux S., Sanders D., 2005, ApJ, 632, 751

Rupke D., Gültekin K., Veilleux S., 2017, ApJ, 850, 40

Santoro F., Rose M., Morganti R., Tadhunter C., Oosterloo T., Holt J., 2018 A\&A, 671, A139

Schnorr-Müller A., Davies R., Korista K., Burtscher L., Rosario D., et al., 2016, MNRAS, 462, 3570

Schnorr-Müller A., Storchi-Bergmann T., Robinson A., Lena D., Nagar N., 2016, MNRAS, 497, 972

Shimizu T., Davies R., Lutz D., Burtscher L., Lin M.-Y., et al., 2019, MNRAS, 490, 5860

Somerville R., Davé R., 2015, ARA\&A, 53, 51

Stasi/'nska G., Vale Asari N., Cid Fernandes R., Gomes J., Schlickmann M., et al., 2008, MNRAS, 391, L29

Stern J., Laor A., 2012, MNRAS, 426, 2703

Storchi-Bergmann T., Simões Lopes R., McGregor P., Riffel R.A., Beck T., Martini P., 2010, MNRAS, 402, 819

Van den Bosch R., Gebhardt K., Gültekin K., Yildirim A., Walsh J., 2015, ApJS, 218, 10

Veilleux S., Osterbrock D., 1987, ApJS, 63, 295

Veilleux S., 1991, ApJ, 369, 331

Veilleux S., Cecil G., Bland-Hawthorn J., 2005, ARA\&A, 43, 769

Veilleux S., Meléndez M., Sturm E., Gracia-Carpio J., Fischer J., et al., 2013, ApJ, 776, 27

Veilleux S., Maiolino R., Bolatto A., Aalto S., 2020, A\&ARv., accepted

Venturi G., Nardini E., Marconi A., Carniani S., Mingozzi M., et al., 2018, A\&A, 619, A74

Wegner G., Bernardi M., Willmer C., da Costa L., Alonso M., et al., 2003, AJ, 126, 2268

Winter L., Veilleux S., McKernan B., Kallman T., 2012, ApJ, 745, 107

\section{APPENDIX A: INDIVIDUAL FITS FOR ACTIVE AND INACTIVE GALAXIES}

Specific fits to the [SII] doublet for one spectrum of each of the active galaxies are shown in Fig. A1. The emission lines were fitted to the reduced spectra presented in Burtscher et al. (2020) before smoothing, in order to retain the full resolution of the dat. The fitting was performed after subtracting a combination of theoretical stellar spectra whose purpose was to minimize the impact of stellar absorption features. During the fit, we have required the profiles of the two lines to be identical, and matched each using two to four Gaussian components (noting that in our analysis, these components are never considered separately). We have checked that the derived uncertainties of the line properties are consistent with the differences between the various spectra for each object before combining them.

Equivalent fits to the [OIII] doublet for the active galaxies are shown in Fig. A2. These have been fitted in the same way as the [SII] doublet, but independently, because the [SII] and $[\mathrm{OIII}]$ profiles are not good matches to each other.

Similar fits for one spectrum of each of the inactive galaxies are shown in Fig. A3. These have been performed 

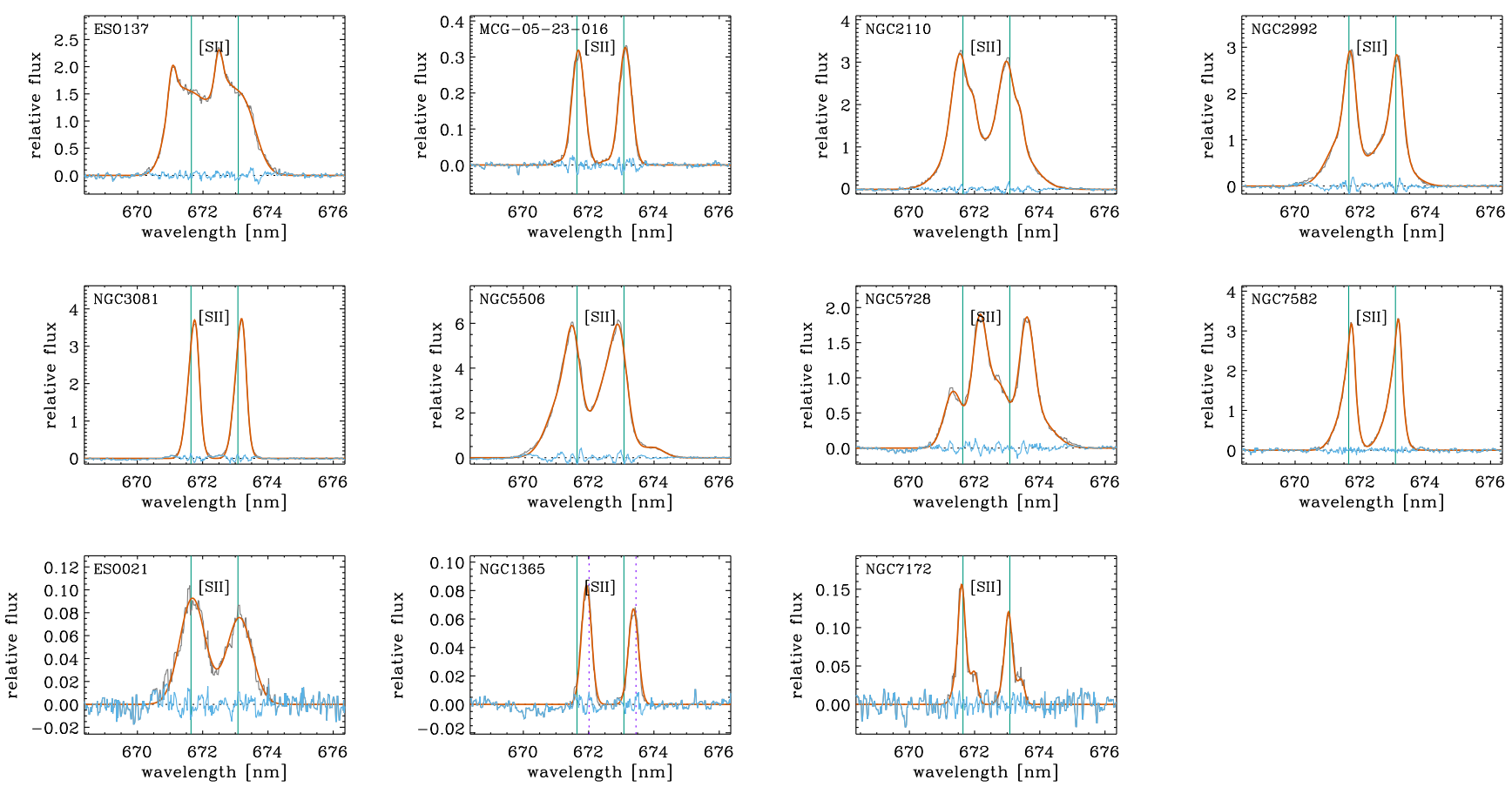

Figure A1. Fits of the [SII] doublet in one spectrum for each active galaxy. These spectra are continuum subtracted, a step that was done before fitting the line profile (see Sec. 2.4).
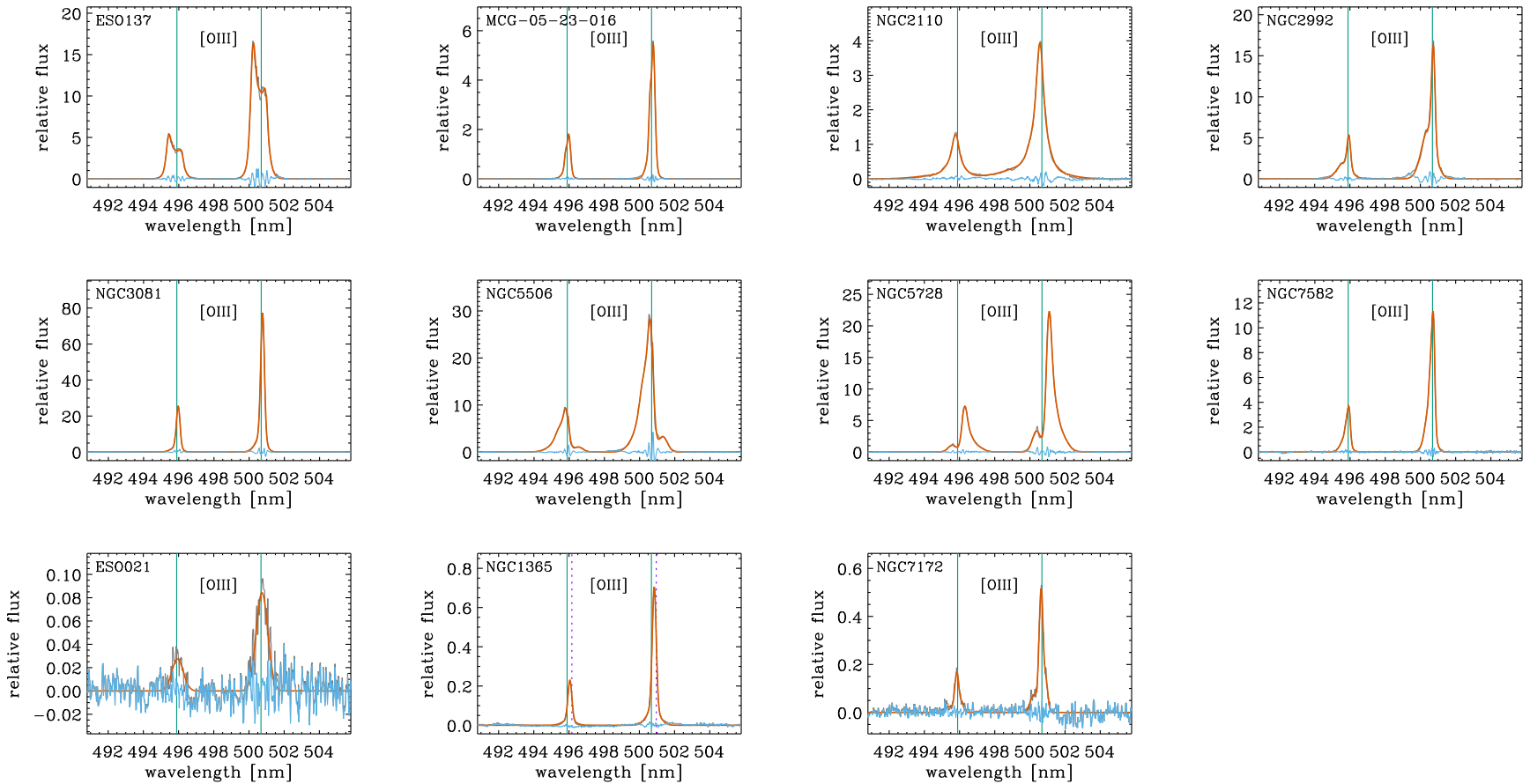

Figure A2. Fits of the [OIII] doublet in one spectrum for each active galaxy, after continuum subtraction. 

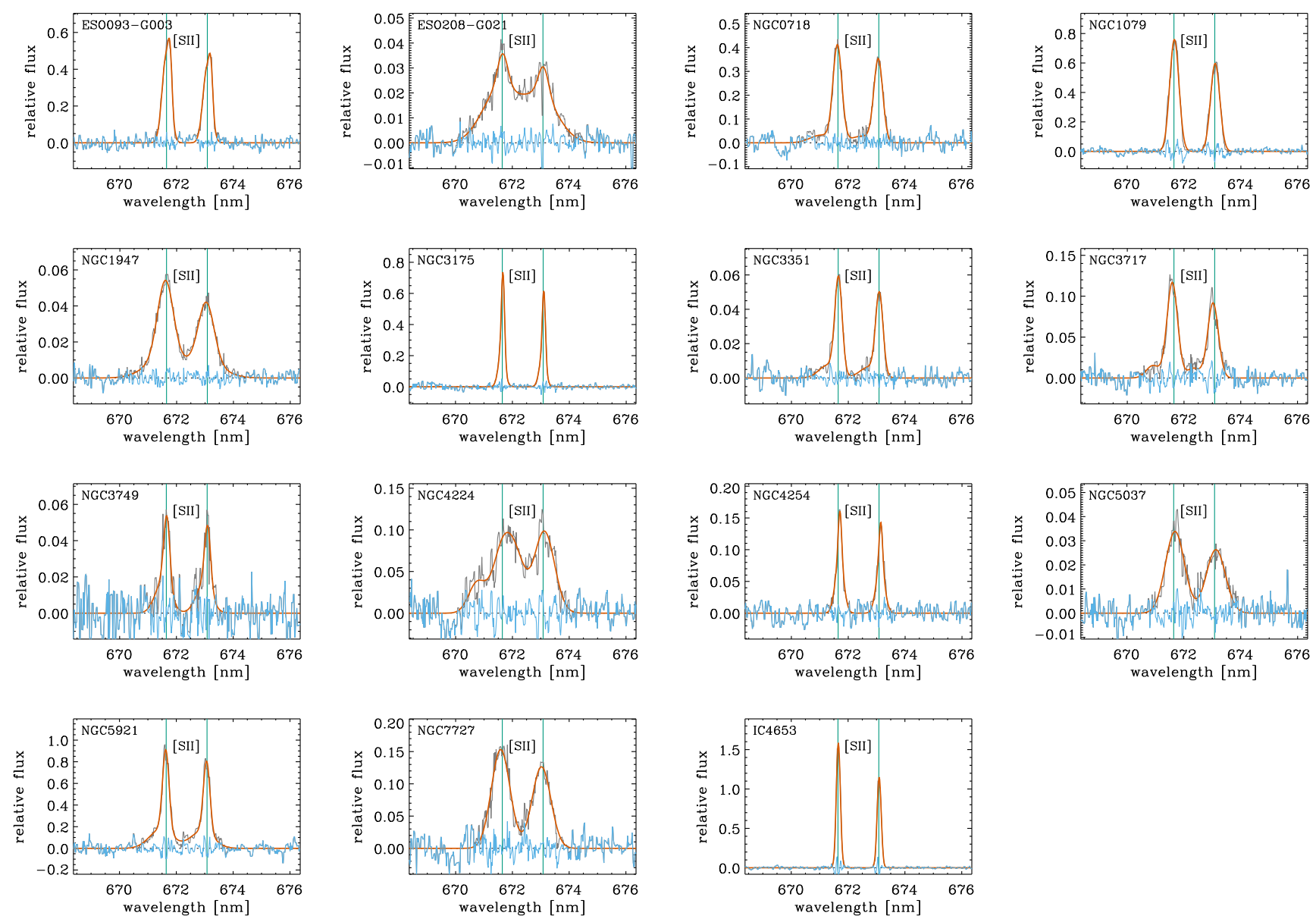

Figure A3. Fits of the [SII] doublet in one spectrum for each inactive galaxy, after continuum subtraction.

in exactly the same way as for the active galaxies, but using only two Gaussian components for each line.

For the eight active galaxies in which we fit the auroral and transauroral lines, the fits to the strong [SII] doublet are repeated in the left-hand panels of Figs. A4 and A5. The remaining three panels in each row of these figures show fits to the other lines used to estimate the electron density in the auroral/transauroral method. The line profiles have been constrained in a similar way to the [SII] doublet, with the additional criterion that only the scaling of each component is allowed to vary (the velocity offset and width are fixed to that derived from the [SII] doublet fit). Especially for the weaker lines, fitting and subtracting the continuum to minimize the impact of stellar absorption features is an important preparatory step. This has been done by fitting theoretical stellar templates to line-free regions over a wider baseline than shown in the panels, as described in Sec. 2.4, and checked by eye. Locations where additional line emission is expected have been marked in the various panels.

The profiles of the $\mathrm{H} \beta$, [SII], and [OIII] lines for the inactive galaxies are shown in Fig. A6. These show a remarkable variety of shapes, although the profiles tend to be centered close to the systemic velocity and asymmetries are less pronounced than for the active galaxies. Although there is a variety of line widths among the objects, they appear to be consistent with the gravitational potential, as indicated by the FWHM of the stellar absorption features which is denoted by the shaded grey region.

This paper has been typeset from a $\mathrm{TEX}_{\mathrm{E}} / \mathrm{LAT}_{\mathrm{EX}}$ file prepared by the author. 

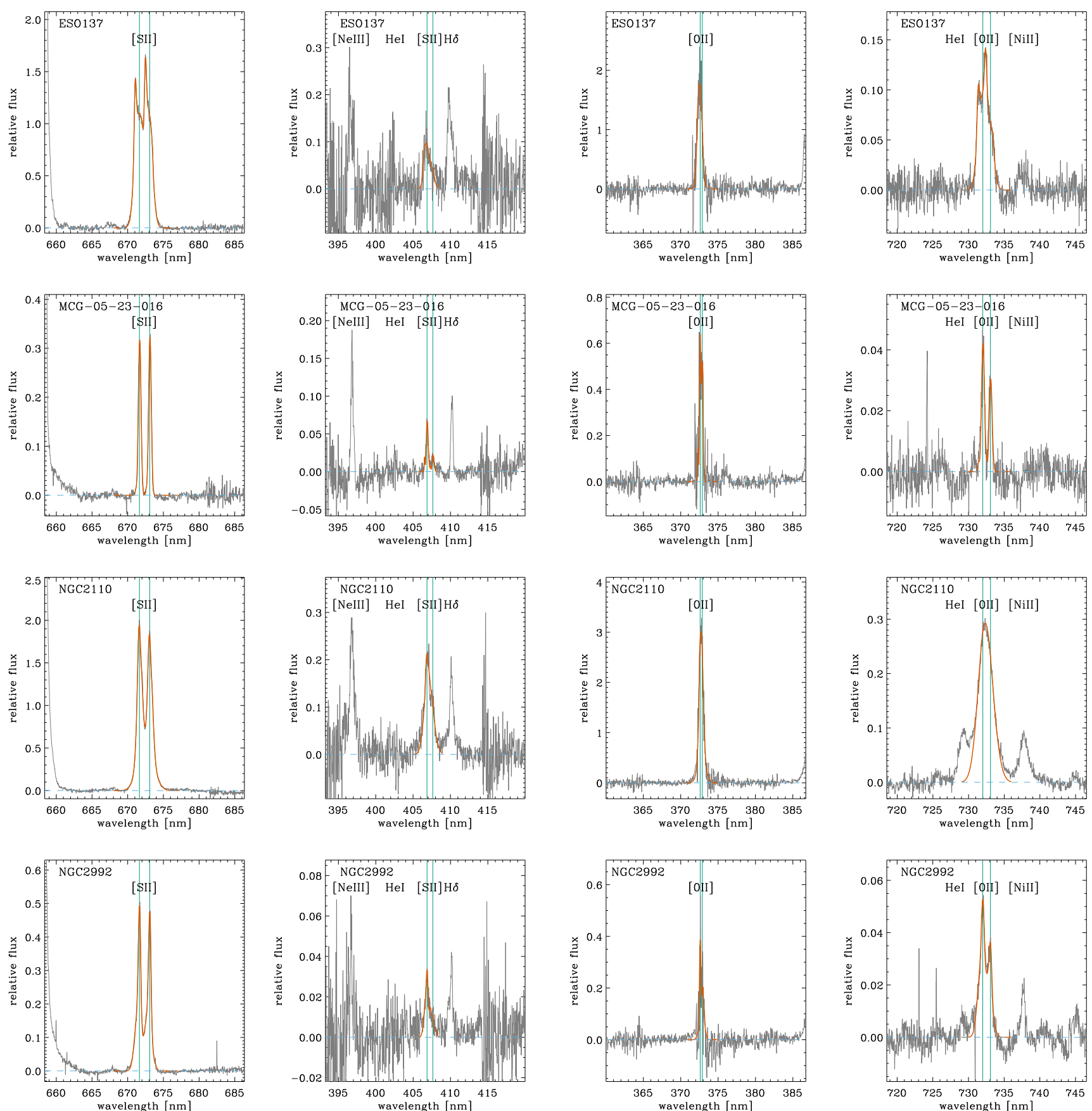

Figure A4. Fits of the various [SII] and [OII] doublets in one spectrum for each active galaxy, after continuum subtraction. 

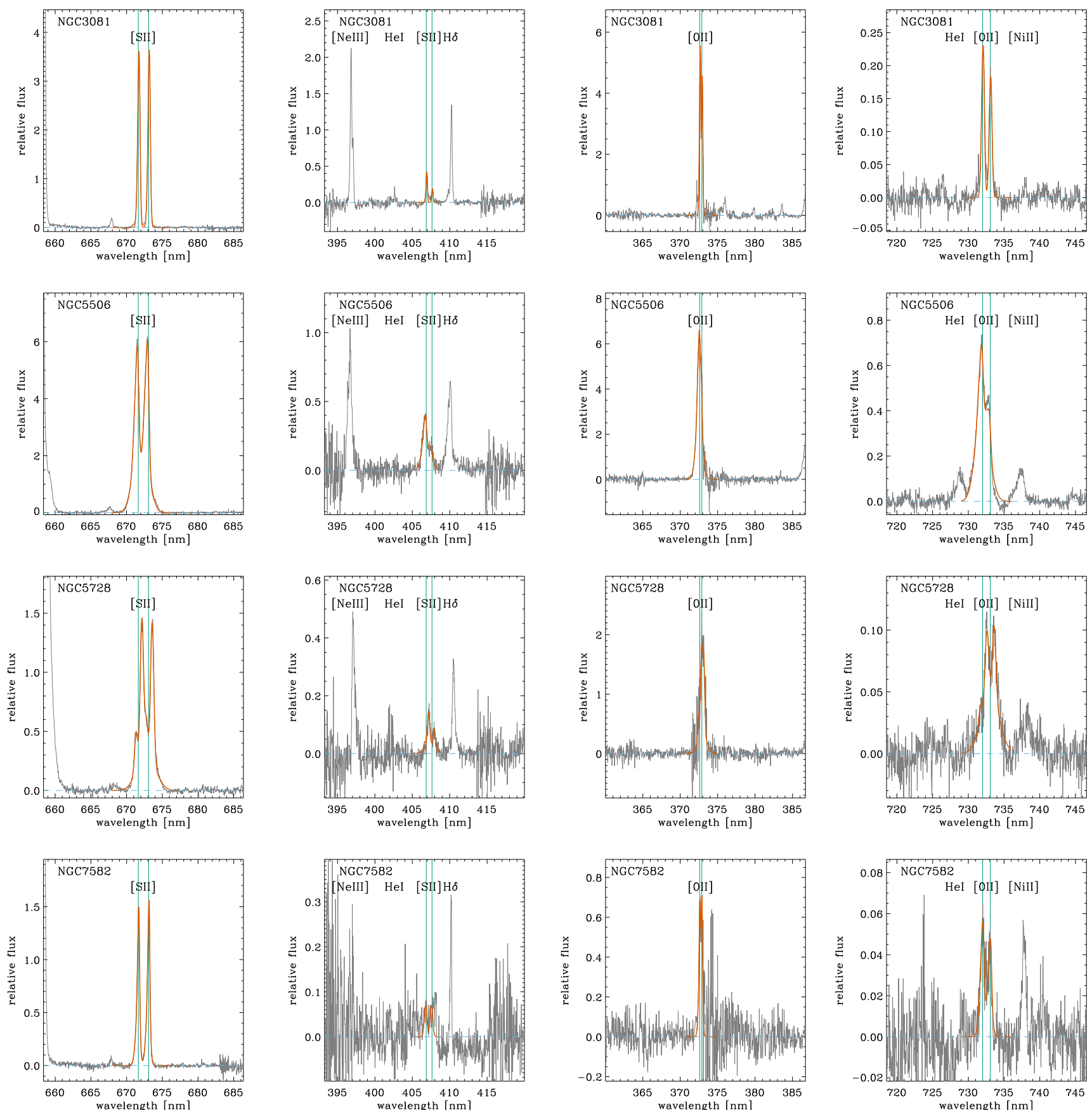

Figure A5. Fits of the various [SII] and [OII] doublets in one spectrum for each active galaxy, after continuum subtraction. 

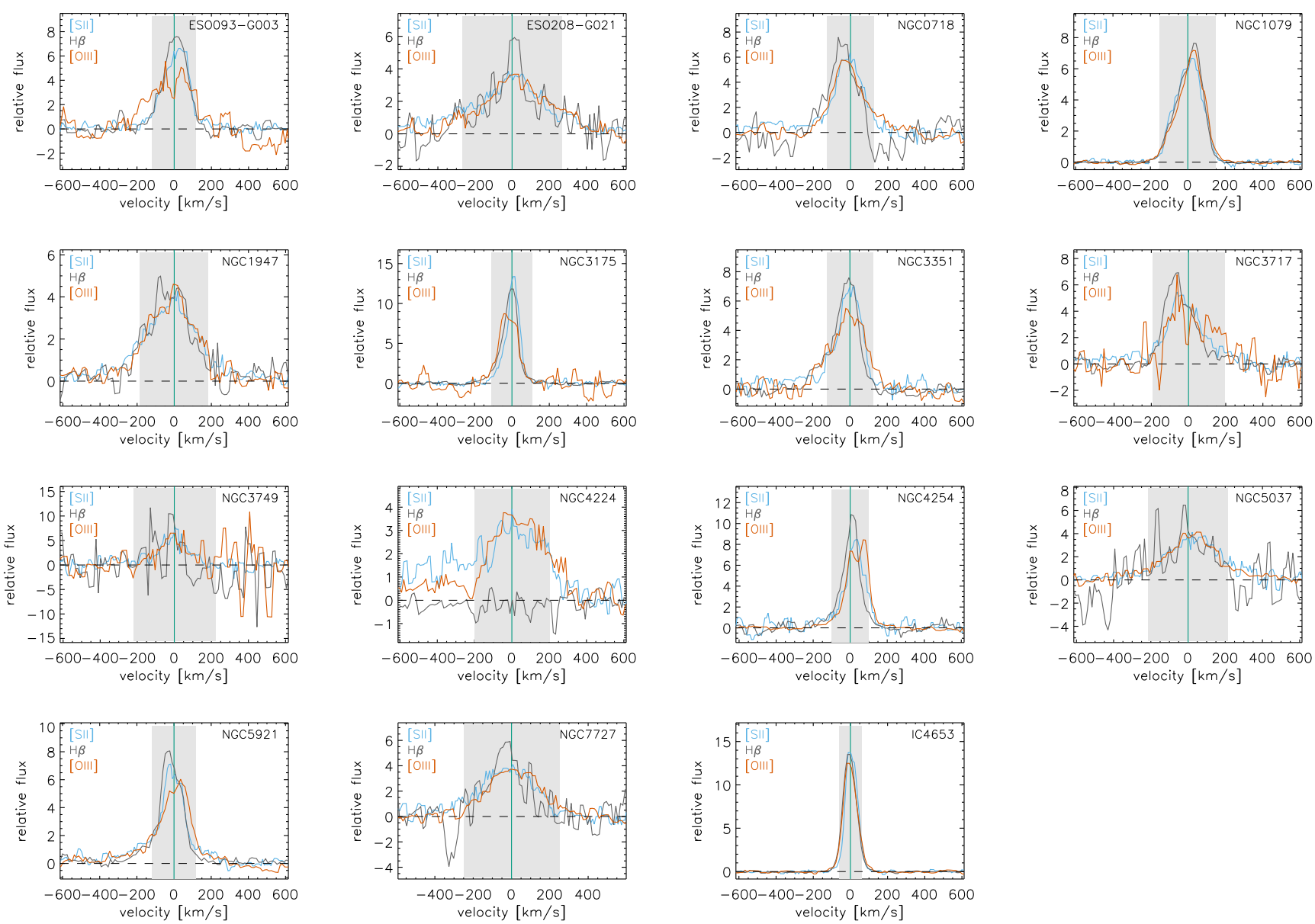

Figure A6. Comparison of the central part of the line profiles of the inactive galaxies as a function of velocity, scaled relatively to the same flux within $\pm 250 \mathrm{~km} \mathrm{~s}^{-1}$. The $\mathrm{H} \beta$, [OIII], and [SII] profiles are shown in grey, red, and blue respectively. For visualisation purposes, the [SII] profile is a combination of the red side of the $6716 \AA$ line and the blue side of the $6731 \AA$ line, scaled to match where they overlap. These profiles show the data only, and not the fits. For reference, the FWHM of the stellar absorption profile is indicated by the shaded grey region. 